\title{
Entanglement of formation and concurrence for mixed states
}

\author{
Xiuhong GAO ${ }^{1}$, Albeverio SERGIO ${ }^{2}$, Kai CHEN ${ }^{3}$, Shaoming FEI $(\bowtie)^{1,2}$, Xianqing LI-JOST ${ }^{4}$ \\ 1 Department of Mathematics, Capital Normal University, Beijing 100037, China \\ 2 Institut für Angewandte Mathematik, Universität Bonn, D-53115, Germany \\ 3 Hefei National Laboratory for Physical Sciences at Microscale, Department of Modern Physics, \\ University of Science and Technology of China, Hefei 230026, China \\ 4 Max Planck Institute for Mathematics in the Sciences, Leipzig D-04103, Germany
}

(c) Higher Education Press and Springer-Verlag 2008

\begin{abstract}
We review some results on analytical computations of the measures for quantum entanglement: entanglement of formation and concurrence. We introduce some estimations of the lower bounds for the entanglement of formation in bipartite mixed states, and of lower bounds for the concurrence in bipartite and tripartite systems. The results on lower bounds for the concurrence are also generalized to arbitrary multipartite systems.
\end{abstract}

Keywords entanglement of formation, concurrence, lower bound

\section{Introduction}

Quantum entanglement plays a crucial role in the rapidly developing theory of quantum information [1], since they constitute the most important resource for quantum information processing. An important theoretical challenge in the theory of quantum entanglement is to give a proper description and quantification of quantum entanglement of multipartite quantum systems. Entanglement of formation (EOF) [2,3-5] and concurrence [6-8] are two well-defined quantitative measures of entanglement. For the two-qubit case, EOF is a monotonically increasing function of the concurrence and an elegant formula for the concurrence was derived analytically by Wootters in Refs. [9,10]. It plays an essential role in describing quantum phase transitions in various interacting quantum many-body systems [11-14] and can be experimentally measured [15].

In the higher dimensional case, due to the extremizations involved in the calculation, only a few explicit analytic formulae for EOF and concurrence have been found for some special symmetric states [16-20]. Some progress,

Received February 2, 2008; accepted March 30, 2008

E-mail: feishm@mail.cnu.edu.cn in particular in the form of practical algorithms, has been obtained on possible lower bounds of the EOF and concurrence for qubit-qudit systems [21-23] and for bipartite systems in arbitrary dimensions [24-26] using numerical optimization over a large number of free parameters. In Refs. [27,28], analytic lower bounds on EOF and concurrence for any dimensional mixed bipartite quantum states have been presented, which have further been shown to be exact for some special classes of states and detect many bound entangled states. In Ref. [29], another lower bound on EOF for bipartite states has been presented from a new separability criterion [30]. A lower bound on concurrence based on a local uncertainty relations (LURs) criterion is obtained in Ref. [31] and this bound is furthermore optimized in Ref. [32].

Although the EOF is only well defined for bipartite systems, the concurrence is well defined even for multipartite states. The lower bound of concurrence for tripartite states has been studied in Ref. [33]. In this review, we first summarize the results related to the analytic formulae and the lower bounds on EOF for bipartite systems, as well as to the lower bounds on concurrence for bipartite and tripartite systems, and then we generalize them to arbitrary multipartite systems.

\section{Entanglement of formation for bipartite systems}

Let $\mathcal{H}_{1}, \mathcal{H}_{2}$ be $N_{1}, N_{2}$-dimensional complex Hilbert spaces with orthonormal basis $e_{i}^{k}, i=1, \ldots, N_{k}, k=1,2$, respectively. A pure quantum state on $\mathcal{H}_{1} \otimes \mathcal{H}_{2}$ is generally of the form,

$$
|\psi\rangle=\sum_{i=1}^{N_{1}} \sum_{j=1}^{N_{2}} a_{i j} e_{i}^{1} \otimes e_{j}^{2}, \quad a_{i j} \in C
$$

with normalization 


$$
\sum_{i=1}^{N_{1}} \sum_{j=1}^{N_{2}} a_{i j} a_{i j}^{*}=1
$$

The EOF $E$ is defined as the partial entropy with respect to the subsystems [34],

$$
\begin{aligned}
E(|\psi\rangle) & =-\operatorname{Tr}\left(\rho_{1} \log _{2} \rho_{1}\right) \\
& =-\operatorname{Tr}\left(\rho_{2} \log _{2} \rho_{2}\right),
\end{aligned}
$$

where $\rho_{1}$ (resp. $\rho_{2}$ ) is the reduced density matrix obtained by tracing $|\psi\rangle\langle\psi|$ (the orthogonal projector onto $|\psi\rangle$ ) over the second (resp. first) Hilbert space of $\mathcal{H}_{1} \otimes \mathcal{H}_{2}$.

It is evident that $E(|\psi\rangle)$ vanishes only for product states. This definition can be extended to mixed states $\rho$ by the convex roof,

$$
E(\rho) \equiv \min _{\left\{p_{i},\left|\psi_{i}\right\rangle\right\}} \sum_{i} p_{i} E\left(\left|\psi_{i}\right\rangle\right)
$$

for all possible ensemble realizations

$$
\rho=\sum_{i} p_{i}\left|\psi_{i}\right\rangle\left\langle\psi_{i}\right|, \quad p_{i} \geqslant 0, \quad \sum_{i} p_{i}=1 .
$$

Consequently, a state $\rho$ is separable if and only if $E(\rho)=0$ and hence can be represented as a convex combination of product states as $\rho=\sum_{i} p_{i} \rho_{i}^{1} \otimes \rho_{i}^{2}$, where $\rho_{i}^{1}$ and $\rho_{i}^{2}$ are pure state density matrices associated to the subsystems $\mathcal{H}_{1}$ and $\mathcal{H}_{2}$, respectively [35]. The measure (4) satisfies all the essential requirements of a good entanglement measure: convexity, no increase under local quantum operations and classical communications on average, no increase under local measurements, asymptotic continuity and other properties [2,3-5].

It is a challenge to calculate Eq. (4) for general mixed states due to the extremizations involved in the calculation. Till now explicit formulae of $E(\rho)$ have been obtained only for a few special cases.

\subsection{EOF for 2-qubits}

In this case, Eq. (3) can be written as $E(|\psi\rangle)=\varepsilon(C(|\psi\rangle))$, where the function $\varepsilon$ is defined by

$$
\begin{aligned}
& \varepsilon(C)=H_{2}\left(\frac{1+\sqrt{1-C^{2}}}{2}\right), \\
& H_{2}(x)=-x \log _{2} x-(1-x) \log _{2}(1-x) .
\end{aligned}
$$

$C$ is called concurrence [9]:

$$
C(|\psi\rangle)=|\langle\psi \mid \tilde{\psi}\rangle|=2\left|a_{11} a_{22}-a_{12} a_{21}\right|,
$$

where $|\tilde{\psi}\rangle=\sigma_{y} \otimes \sigma_{y}\left|\psi^{*}\right\rangle,\left|\psi^{*}\right\rangle$ is the complex conjugate of $|\psi\rangle, \sigma_{y}$ is the Pauli matrix, $\sigma_{y}=\left(\begin{array}{cc}0 & -i \\ i & 0\end{array}\right)$.
As $E$ is a monotonically increasing function of $C, C$ can be also taken as a kind of measure of entanglement. Calculating the minimum in Eq. (4) is reduced to calculating the corresponding minimum of

$$
C(\rho)=\min _{\left\{p_{i},\left|\psi_{i}\right\rangle\right\}} \sum_{i=1} p_{i} C\left(\left|\psi_{i}\right\rangle\right),
$$

which simplifies the computation.

The formula for the entanglement of a pair of qubits in any mixed states $\rho$ is given in Ref. [10]: $E(\rho)=\varepsilon(C(\rho))$, with $C(\rho)=\max \left(0, \lambda_{1}-\lambda_{2}-\lambda_{3}-\lambda_{4}\right)$, here the $\lambda_{i}^{\prime} \mathrm{s}$ are the square roots of the eigenvalues of $\rho\left(\sigma_{y} \otimes \sigma_{y}\right)$ $\cdot \rho^{*}\left(\sigma_{y} \otimes \sigma_{y}\right)$ in descending order. The concurrence is itself a measure of entanglement that provides an analytic formula for the EOF for a pair of qubits.

The direct experimental measurement of $C(|\psi\rangle)$ is not possible due to the unphysical operation of the complex conjugation in Eq. (6). Nevertheless, it has been shown that any $m$-th degree polynomial function of a density matrix $\rho$ can be measured on an $m$-fold copy of $\rho$ [36]. By considering a twofold copy of the state in question, the concurrence $C$ of an arbitrary state $|\psi\rangle$ can be defined as $C=2 \sqrt{P_{A}}$, where $P_{A}=\langle\psi|\otimes\langle\psi|A| \psi\rangle \otimes| \psi\rangle$ is the probability of observing the two copies of the first subsystem in an antisymmetric state, that is, a state that acquires a phase shift of $\pi$ upon exchange of the constituents, and $A$ is the corresponding measurement operator [15].

\subsection{EOF for isotropic states}

The EOF for a class of mixed states in arbitrary dimension $N_{1}=N_{2}=N$, the isotropic states, was presented by Terhal and Vollbrecht [16]. The isotropic states are invariant under the transformations $U \otimes U^{*}$, for any unitary transformation $U$. They have the form

$$
\rho_{F}=\frac{1-F}{N^{2}-1}\left(I-\left|\Psi^{+}\right\rangle\left\langle\Psi^{+}\right|\right)+F\left|\Psi^{+}\right\rangle\left\langle\Psi^{+}\right|,
$$

where $\left|\Psi^{+}\right\rangle \equiv \sqrt{1 / N} \sum_{i=1}^{N}|i i\rangle$

$F=\left\langle\Psi^{+}\left|\rho_{F}\right| \Psi^{+}\right\rangle, 0 \leqslant F \leqslant 1$, is the fidelity of $\rho_{F}$. It is shown that for $F \geqslant 1 / N$, the EOF for isotropic states is $E\left(\rho_{F}\right)=\operatorname{co}[R(F)]$, where $R(F)$ is a simple function of $F$, and "co" stands for the convex hull. That is the largest convex function bounded above by the given function. For $N=2,3$, the EOF for $\rho_{F}$ is given by

$$
E\left(\rho_{F}\right)= \begin{cases}0, & F \leqslant \frac{1}{N}, \\ R_{1, N-1}(F), & F \in\left(\frac{1}{N}, \frac{4(N-1)}{N^{2}}\right), \\ \frac{N \log _{2}(N-1)}{N-2}(F-1)+\log _{2} N, & F \in\left[\frac{4(N-1)}{N^{2}}, 1\right],\end{cases}
$$


where

$$
R_{1, N-1}(F)=H_{2}(\gamma(F))+(1-\gamma(F)) \log _{2}(N-1),
$$

with

$$
\gamma(F)=\frac{1}{N}(\sqrt{F}+\sqrt{(N-1)(1-F)})^{2} .
$$

For general $N$, the correctness of this formula is proved in Ref. [38].

\subsection{EOF for Werner states}

Werner states are a class of mixed states for $N \times N$ systems which are invariant under the transformations $U \otimes U$ for any unitary transformation $U[35,39]$. The density matrix of these states can be expressed as

$$
\rho_{f}=\frac{1}{N^{3}-N}(N-f) I+(N f-1) P,
$$

where $P$ is the flip operator (or swap operator) defined by $P(\phi \otimes \psi)=\psi \otimes \phi$. In the computational basis $|i j\rangle, P$ is of the form $P=\sum_{i, j}^{N}|i j\rangle\langle j i|$. Here, $f$ is a constant $f=\langle P\rangle \equiv \operatorname{Tr}\left(P \rho_{f}\right)$ satisfying $-1 \leqslant f \leqslant 1$. Werner states are separable if and only if $f \geqslant 0$, as shown in Refs. $[35,39]$.

The EOF of Werner states has been derived from an extremization procedure [39],

$$
E\left(\rho_{f}\right)=H_{2}\left(\frac{1}{2}\left(1-\sqrt{1-f^{2}}\right)\right) .
$$

Since $E\left(\rho_{f}\right)$ is a monotonically increasing function of $-f$, as seen from Eq. (13), it is expected [40] that $-f$ plays the role of concurrence, similarly as in the two qubits case [10].

Instead of Eq. (6), the generalized concurrence for a pure state $|\psi\rangle$ in the tensor space $\mathcal{H}_{1} \otimes \mathcal{H}_{2}$ is defined by Refs. [6-8],

$$
C(|\psi\rangle)=\sqrt{2\left(1-\operatorname{Tr} \rho_{1}^{2}\right)},
$$

where $\rho_{1}$ is the reduced density matrix. The concurrence (7) of Werner states is given by Ref. [41]

$$
C\left(\rho_{f}\right)= \begin{cases}|f|=-f, & \text { for } f<0, \\ 0, & \text { for } f \geqslant 0 .\end{cases}
$$

This shows that the EOF of Werner states is a monotonically increasing function of the concurrence. Namely, the conjecture [40] that $-f$ plays exactly the role of concurrence is verified. Furthermore, it is shown that the concurrence and EOF of Werner states have the same optimal decomposition [41].

\subsection{EOF for a special class of mixed states}

For $N_{1}=N_{2}=N \geqslant 3$, there is no such concurrence $C$ that entanglement of formation $E$ as given by Eq. (4) is a monotonically increasing function of $C$. The concurrences discussed in Ref. [6-8] can be only used to judge whether a pure state is separable (or maximally entangled) or not $[42,43]$. The EOF is no longer a monotonically increasing function of these concurrences. Nevertheless, if one considers special classes of quantum states, certain quantities (generalized concurrence) can be found to simplify the calculation of the corresponding EOF [17].

Let $A$ denote the matrix with entries given by $a_{i j}$ in Eq. (1), $i, j=1, \ldots, N$. The reduced density matrix $\rho_{1}$ can be expressed as

$$
\rho_{1}=A A^{\dagger} .
$$

If $A A^{\dagger}$ has only two non-zero eigenvalues $\lambda_{1}$ (resp. $\lambda_{2}$ ) with degeneracy $n$ (resp. $m$ ), $n+m \leqslant N$, we denote $\mathcal{D}$ the maximal non-zero diagonal determinant

$$
\mathcal{D}=\lambda_{1}^{n} \lambda_{2}^{m}
$$

From the normalization of $|\psi\rangle$, one has $\operatorname{Tr}\left(A A^{\dagger}\right)=1$, i.e.,

$$
n \lambda_{1}+m \lambda_{2}=1
$$

$\lambda_{1}$ (resp. $\left.\lambda_{2}\right)$ takes values $\left(0, \frac{1}{n}\right)\left(\right.$ resp. $\left.\left(0, \frac{1}{m}\right)\right)$. In this case the EOF of $|\psi\rangle$ is given by

$$
E(|\psi\rangle)=-n \lambda_{1} \log _{2} \lambda_{1}-m \lambda_{2} \log _{2} \lambda_{2} .
$$

According to (17) and (18), one has

$$
\frac{\partial E}{\partial \mathcal{D}}=\frac{m \lambda_{1}^{1-n}}{1-n \lambda_{1}-m \lambda_{1}}\left(\frac{1-n \lambda_{1}}{m}\right)^{1-m} \log _{2} \frac{1-n \lambda_{1}}{m \lambda_{1}},
$$

which is positive for $\lambda_{1} \in\left(0, \frac{1}{n}\right)$. Therefore, $E(|\psi\rangle)$ is a monotonically increasing function of $\mathcal{D}$. $\mathcal{D}$ is a generalized concurrence and can be taken as a kind of measure of entanglement in this case.

From Eqs. (18) and (19), the quantum states with the measure of entanglement characterized by $\mathcal{D}$ are generally entangled. They are separated when $n=1, \lambda_{1} \rightarrow 1$ $\left(\lambda_{2} \rightarrow 0\right)$ or $m=1, \lambda_{2} \rightarrow 1\left(\lambda_{1} \rightarrow 0\right)$. For the case $n=m>1$, all the pure states in this class are non-separable. In this case,

$$
E(|\psi\rangle)=n\left(-x \log _{2} x-\left(\frac{1}{n}-x\right) \log _{2}\left(\frac{1}{n}-x\right)\right),
$$

where $x=\frac{1}{2}\left(\frac{1}{n}+\sqrt{\frac{1}{n^{2}}\left(1-d^{2}\right)}\right)$ and 


$$
d \equiv 2 n \mathcal{D}^{\frac{1}{2 n}}=2 n \sqrt{\lambda_{1} \lambda_{2}} .
$$

The generalized concurrence $d$ takes values in $[0,1]$. From (21) one can show that $E(d)$ is a convex function. Instead of calculating $E(\rho)$ directly, one may calculate the minimum decomposition of $\mathcal{D}(\rho)$ or $d(\rho)$ to simplify the calculations.

Consider a class of pure states (1) with the matrix $A$ given by

$$
A=\left(\begin{array}{cccc}
0 & b & a_{1} & b_{1} \\
-b & 0 & c_{1} & d_{1} \\
a_{1} & c_{1} & 0 & -e \\
b_{1} & d_{1} & e & 0
\end{array}\right)
$$

$a_{1}, b_{1}, c_{1}, d_{1}, b, e \in \mathbb{C}$. The matrix $A A^{\dagger}$ has two eigenvalues with degeneracy two, i.e., $n=m=2$ and $\left|A A^{\dagger}\right|=$ $\left|b_{1} c_{1}-a_{1} d_{1}+b e\right|^{4}$. The generalized concurrence $d$ is given by $d=4\left|b_{1} c_{1}-a_{1} d_{1}+b e\right|$.

Let $p$ be a $16 \times 16$ matrix with only non-zero entries $p_{1,16}=p_{2,15}=-p_{3,14}=p_{4,10}=p_{5,12}=p_{6,11}=p_{7,13}=-p_{8,8}$ $=-p_{9,9}=p_{10,4}=p_{11,6}=p_{12,5}=p_{13,7}=-p_{14,3}=p_{15,2}=p_{16,1}$ $=1$. $d$ can be further written as

$$
d=\left|\left\langle\psi \mid p \psi^{*}\right\rangle\right|
$$

Let $\boldsymbol{\Psi}$ denote the set of pure states (1) with $A$ of form (23). Consider all mixed states with density matrix $\rho$ such that its decompositions are of the form

$$
\rho=\sum_{i=1}^{M} p_{i}\left|\psi_{i}\right\rangle\left\langle\psi_{i}\left|, \quad \sum_{i=1}^{M} p_{i}=1, \quad\right| \psi_{i}\right\rangle \in \Psi .
$$

All other kind of decompositions, (say decomposition with respect to $\left.\left|\psi_{i}^{\prime}\right\rangle \neq|\psi\rangle\right)\left|\psi_{i}^{\prime}\right\rangle$, can be obtained from a unitary linear combination of $\left|\psi_{i}\right\rangle[9,17]$. As linear combinations of $\left|\psi_{i}\right\rangle$ do not change the form of the corresponding matrices (23), once $\rho$ has a decomposition with respect to $\left|\psi_{i}\right\rangle \in \Psi$, all other decompositions $\left|\psi_{i}^{\prime}\right\rangle$, including the minimum decomposition of the EOF, also satisfy $\left|\psi_{i}^{\prime}\right\rangle \in \Psi$. Then the minimum decomposition of the generalized concurrence is Ref. [17]

$$
d(\rho)=\Lambda_{1}-\sum_{i=2}^{16} \Lambda_{i}
$$

where $\Lambda_{i}$, in decreasing order, are the eigenvalues of the Hermitian matrix $R \equiv \sqrt{\sqrt{\rho} p \rho^{*} p \sqrt{\rho}}$, or, alternatively, the square roots of the eigenvalues of the non-Hermitian matrix $\rho p \rho^{*} p$.

An important fact used in the derivation of (26) is that the generalized concurrence $d$ is a quadratic form of the entries of the matrix $A$, so that $d$ can be expressed in the form of (24) in terms of a suitable matrix $p$. An $N$-dimensional pure state (1) is called $d$-computable if $A$ satisfies the following relations:

$$
\begin{aligned}
& \left|A A^{\dagger}\right|=\left([A][A]^{*}\right)^{N / 2}, \\
& \left|A A^{\dagger}-\lambda I d_{N}\right|=\left(\lambda^{2}-\|A\| \lambda+[A][A]^{*}\right)^{N / 2},
\end{aligned}
$$

where $[A]$ and $\|A\|$ are quadratic forms of $a_{i j}$, and $I d_{N}$ is the $N \times N$ identity matrix. Let $\mathcal{A}$ be the set of matrices satisfying (27), which implies that for $A \in \mathcal{A}, A A^{\dagger}$ has at most two different eigenvalues, each one of which has order $N /$ 2. $d$ is a quadratic form of the entries of the matrix $A$.

$N$-dimensional, $N=2^{k}, 2 \leqslant k \in \mathbb{N}, d$-computable states can be constructed as follows [18]. Set $A_{2}=\left(\begin{array}{cc}a & -c \\ c & d\end{array}\right)$, where $a, c, d \in \mathbb{C}$. For any $b_{1}, c_{1} \in \mathbb{C}$, a $4 \times 4$ matrix $A_{4} \in \mathcal{A}$ can be constructed in the following way,

$$
A_{4}=\left(\begin{array}{cc}
B_{2} & A_{2} \\
-A_{2}^{t} & C_{2}^{t}
\end{array}\right)
$$

where

$$
B_{2}=b_{1} J_{2}, \quad C_{2}=c_{1} J_{2}, \quad J_{2}=\left(\begin{array}{cc}
0 & 1 \\
-1 & 0
\end{array}\right),
$$

where $t$ stands for transpose. It is straightforward to verify that $A_{4}$ satisfies the relations in (27).

For general construction of high dimensional matrices $A_{2^{k+1}} \in \mathcal{A}, 2 \leqslant k \in \mathbb{N}$, one has

$$
\begin{gathered}
A_{2^{k+1}}=\left(\begin{array}{cc}
B_{2^{k}} & A_{2^{k}} \\
(-1)^{\frac{k(k+1)}{2}} A_{2^{k}}^{t} & C_{2^{k}}^{t}
\end{array}\right), \\
J_{2^{k+1}}=\left(\begin{array}{cc}
0 & J_{2^{k}} \\
(-1)^{\frac{(k+1)(k+2)}{2}} J_{2^{k}}^{t} & 0
\end{array}\right),
\end{gathered}
$$

where $B_{2^{k}}=b_{k} J_{2^{k}}, C_{2^{k}}=c_{k} J_{2^{k}}, b_{k}, c_{k} \in \mathbb{C} . J_{2^{k+1}}$ are called multipliers.

For all $N^{2} \times N^{2}$ density matrices with decompositions on these $N$-dimensional $d$-computable pure states, their EOF can be calculated by formulae similar to (26).

The results can be generalized to the case that $A A^{\dagger}$ has $n \geqslant 3$ different non-zero eigenvalues [19]. Let $\lambda_{1}, \lambda_{2}, \ldots, \lambda_{n}$, each with degeneracy $m, m n \leqslant N$, be the non-zero eigenvalues of $A A^{\dagger} . \lambda_{i}=\lambda_{i}(u, v), i=1,2, \ldots, n$, are differentiable functions of two real variables $u$ and $v$. Define $D=m n \sqrt{\lambda_{1} \lambda_{2} \cdots \lambda_{n}}$. If $\lambda_{i}=\lambda_{i}(u, v), i=1,2, \ldots, n$, satisfy

$$
\sum_{i} \frac{\partial \lambda_{i}}{\partial D} \log _{2} \lambda_{i}<0
$$

then $D$ is a measure of entanglement in the sense that the EOF of the corresponding pure state is a monotonically increasing function of $D$. 
As an example, consider the non-zero eigenvalues of $A A^{\dagger}$ to be $\lambda_{1}=u, \lambda_{2}=u+v, \lambda_{3}=u+2 v$, each with degeneracy $m, u$ and $v \in \mathbb{R}$ taking values in $\left(0, \frac{1}{3 m}\right)$. The generalized concurrence is given by $D=3 m \sqrt{u(u+v)(u+2 v)}$. It is straightforward to verify that $E$ is a monotonically increasing function of $D$, since

$\sum_{i} \frac{\partial \lambda_{i}}{\partial D} \log _{2} \lambda_{i}=\frac{1}{3 m v \sqrt{3 m}}\left(1-9 m^{2} v^{2}\right)^{1 / 2} \log _{2} \frac{1-3 m v}{1+3 m v}<0$.

Due to the relation

$$
\begin{aligned}
& \sum_{i} \frac{1}{\lambda_{i}}\left(\frac{\partial \lambda_{i}}{\partial D}\right)^{2}+\frac{\partial^{2} \lambda_{i}}{\partial D^{2}} \ln \lambda_{i} \\
& =\frac{1}{27 m^{3} v^{3}}\left(6 m v+\ln \frac{1-3 m v}{1+3 m v}\right)<0,
\end{aligned}
$$

$E$ is also a convex function of $D$.

As $E(|\psi\rangle)$ is a monotonically increasing and convex function of $D$, instead of calculating $E(\rho)$, one may calculate the minimum decomposition (in the sense of Eq. (4)),

$$
D(\rho)=\min \sum_{a=1}^{M} p_{a} D\left(\left|\psi_{a}\right\rangle\right),
$$

to simplify the calculations, as long as $\rho$ has all decompositions on pure states with their eigenvalues of $A A^{\dagger}$ satisfying (31). Nevertheless, like $E(|\psi\rangle)$, generally the expression of $D(|\psi\rangle)=m n \sqrt{\lambda_{1} \lambda_{2} \cdots \lambda_{n}}$ can still be quite complicated.

If the generalized concurrence $D=m n \sqrt{\lambda_{1} \lambda_{2} \cdots \lambda_{n}}$ satisfying (31) can be further expressed as

$$
D=\frac{m n}{\sqrt{2}} \sqrt{I_{0}^{2}-I_{1}},
$$

where

$$
\begin{aligned}
& I_{0}=\operatorname{Tr}\left(A A^{\dagger}\right)=\sum_{i, j=1}^{N} a_{i j} a_{i j}^{*}, \\
& I_{1}=\operatorname{Tr}\left[\left(A A^{\dagger}\right)^{2}\right]=\sum_{i, j, p, q=1}^{N} a_{i p} a_{i q}^{*} a_{j q} a_{j p}^{*},
\end{aligned}
$$

the calculation of the corresponding EOF is then greatly simplified.

Let $\Psi$ denote the set of all pure states of the form (1) such that

i) (31) is satisfied;

ii) the EOF is a convex function of $D$, i.e., $\sum_{i} \frac{1}{\lambda_{i}}\left(\frac{\partial \lambda_{i}}{\partial D}\right)^{2}+\frac{\partial^{2} \lambda_{i}}{\partial D^{2}} \ln \lambda_{i}<0$

iii) $D=m n \sqrt{\lambda_{1} \lambda_{2} \cdots \lambda_{n}}=\frac{m n}{\sqrt{2}} \sqrt{I_{0}^{2}-I_{1}}$.

A mixed state $\rho$ given by (5) is called $D$-computable if all the decompositions of $\rho$ into pure states belong to $\Psi$.

Due to the conditions i) and ii), for a $D$-computable state $\rho$, calculating $E(\rho)$ is then reduced to the calculation of the corresponding minimum of

$$
D(\rho)=\min \sum_{a=1}^{M} p_{a} D\left(\left|\psi_{a}\right\rangle\right),
$$

which simplifies the calculation if $D\left(\left|\psi_{a}\right\rangle\right)$ has a simpler expression than $E\left(\left|\psi_{a}\right\rangle\right)$. The condition iii) guarantees that $D$ is a quadratic form of the entries of the matrix $A$ and can be expressed as $D=\left|\left\langle\psi \mid S \psi^{*}\right\rangle\right|$ in terms of a suitable matrix $S$, which allows one to find an explicit analytical expression of the EOF in a way similar to the one used in Refs. [9] and [17].

Let $S^{i j i q}$ be a symmetric $N^{2} \times N^{2}$ matrix whose elements are all zero except for

$$
\begin{aligned}
& S_{p+N(i-1), q+N(j-1)}=S_{q+N(j-1), p+N(i-1)}=1, \\
& S_{q+N(i-1), p+N(j-1)}=S_{p+N(j-1), q+N(i-1)}=-1,
\end{aligned}
$$

where $i, j, p, q=1, \ldots, N$. Let $\Lambda_{1}^{i p j q}, \Lambda_{2}^{i p j q}, \Lambda_{3}^{i p j q}$ and $\Lambda_{4}^{i p j q}$, in decreasing order, be the eigenvalues of the rank four Hermitian matrix $\sqrt{\sqrt{\rho} S^{i p j q} \rho^{*} S^{i p j q} \sqrt{\rho}}$.

For a $D$-computable state $\rho$, the minimum decomposition of the generalized concurrence $D(\rho)$, i.e., the average generalized concurrence of the pure states of the decomposition, minimized over all decompositions of $\rho$, is given by

$$
\frac{m n}{4}\left[\sum_{i, j, p, q=1}^{N}\left(\Lambda_{1}^{i p j q}-\Lambda_{2}^{i p j q}-\Lambda_{3}^{i p j q}-\Lambda_{4}^{i p j q}\right)^{2}\right]^{1 / 2} .
$$

Due to the convex relation between $E(|\psi\rangle)$ and $D(|\psi\rangle)$, the EOF of $\rho$ is given by $E(D(\rho))$.

\section{Lower bounds of EOF and concurrence for mixed states}

It is generally difficult to calculate the minimum (4) for arbitrarily given (5). Instead of finding the exact minimum, one may also try to find the lower bound of EOF or concurrence.

\subsection{Lower bounds of EOF for bipartite mixed states}

Let $\mathcal{H}_{1}, \mathcal{H}_{2}$ be $N_{1}, N_{2}$-dimensional $\left(N_{1} \leqslant N_{2}\right)$ Hilbert spaces, respectively. A pure state $|\psi\rangle$ in $\mathcal{H}_{1} \otimes \mathcal{H}_{2}$ has a standard Schmidt form

$$
|\psi\rangle=\sum_{i}^{N_{1}} \sqrt{\mu_{i}}\left|a_{i} b_{i}\right\rangle
$$

where $\sqrt{\mu_{i}}, i=1, \ldots, N_{1}$, are the Schmidt coefficients, $\left|a_{i}\right\rangle$ and $\left|b_{i}\right\rangle$ are orthonormal basis in $\mathcal{H}_{1}$ and $\mathcal{H}_{2}$, respectively.

From (3), the EOF for $|\psi\rangle$ is given by 


$$
E(|\psi\rangle)=-\sum_{i=1}^{N_{1}} \mu_{i} \log _{2} \mu_{i}=H(\vec{\mu}),
$$

where $\vec{\mu}$ is the Schmidt vector $\left(\mu_{1}, \mu_{2}, \ldots, \mu_{N_{1}}\right)$.

Let $\|G\|$ denote the trace norm of a matrix $G$ defined by $\|G\|=\operatorname{Tr}\left(G G^{\dagger}\right)^{1 / 2}$. Set $\rho=|\psi\rangle\langle\psi|$. It is easy to see that

$$
\left\|\rho^{T_{1}}\right\|=\|\mathcal{R}(\rho)\|=\left(\sum_{i}^{N_{1}} \sqrt{\mu_{i}}\right)^{2} \equiv \lambda,
$$

where $\rho^{T_{1}}$ is the partial transposed matrix of $\rho$ with respect to the first subsystem, $\mathcal{R}(\rho)$ is the realigned matrix of $\rho$ defined by $\mathcal{R}(\rho)_{i j, k l}=\rho_{i k, j l}$, where $i$ and $j$ are the row and column indices with respect to the first subsystem respectively, while $k$ and $l$ are the corresponding indices for the second subsystem [44-46].

Assume that one has already found an optimal decomposition $\Sigma_{i} p_{i} \rho^{i}$ for $\rho$ to achieve the infimum of $E(\rho)$, where $\rho^{i}$ are pure state density matrices. Then $E$ $(\rho)=\Sigma_{i} p_{i} E\left(\rho^{i}\right)$ by definition. For a given $\lambda, H(\vec{\mu})$ in (34) has a minimum [16],

$$
\begin{aligned}
R(\lambda) & =\min _{\vec{\mu}}\left\{H(\vec{\mu}) \mid\left(\sum_{k=1}^{N_{1}} \sqrt{\mu_{k}}\right)^{2}=\lambda\right\} \\
& =H_{2}[\gamma(\lambda)]+[1-\gamma(\lambda)] \log _{2}\left(N_{1}-1\right),
\end{aligned}
$$

where

$$
\gamma(\lambda)=\frac{1}{N_{1}^{2}}\left[\sqrt{\lambda}+\sqrt{\left(N_{1}-1\right)\left(N_{1}-\lambda\right)}\right]^{2} .
$$

Moreover, $\operatorname{co}[R(\lambda)]$ is a monotonously increasing, convex function and satisfies $\operatorname{co}[R(\lambda)] \leqslant H(\vec{\mu})$ for a given $\lambda$. Set $\mathcal{E}(\lambda)=\operatorname{co}[R(\lambda)]$, then one thus has

$$
\begin{aligned}
E(\rho) & =\sum_{i} p_{i} E\left(\rho^{i}\right)=\sum_{i} p_{i} H\left(\vec{\mu}^{i}\right) \\
& \geqslant \sum_{i} p_{i} \varepsilon\left(\lambda^{i}\right) \geqslant \varepsilon\left(\sum_{i} p_{i} \lambda^{i}\right) \\
& \geqslant\left\{\begin{array}{l}
\varepsilon\left(\left\|\rho^{T_{A}}\right\|\right), \\
\varepsilon(\|\mathcal{R}(\rho)\|),
\end{array}\right.
\end{aligned}
$$

where the monotonicity and convexity properties of $\mathcal{E}$, and convexity of the trace norm

$$
\begin{aligned}
& \left\|\rho^{T_{A}}\right\| \leqslant \sum_{i} p_{i}\left\|\left(\rho^{i}\right)^{T_{A}}\right\|, \\
& \|\mathcal{R}(\rho)\| \leqslant \sum_{i} p_{i}\left\|\mathcal{R}\left(\rho^{i}\right)\right\|,
\end{aligned}
$$

have been used. Setting $\Lambda=\max \left[\left\|\rho^{T_{A}}\right\|,\|\mathcal{R}(\rho)\|\right]$, one obtains

$$
E(\rho) \geqslant \varepsilon(\Lambda)=c o[R(\Lambda)] .
$$

If the function $R(\Lambda)$ has only one reflection point, $\operatorname{co}[R(\Lambda)]$ can be obtained explicitly from $R(\Lambda)$ [16]. One has then

$E(\rho) \geqslant \begin{cases}0, & \Lambda=1, \\ H_{2}[\gamma(\Lambda)]+[1-\gamma(\Lambda)] \log _{2}\left(N_{1}-1\right), & \Lambda \in\left[1, \frac{4\left(N_{1}-1\right)}{N_{1}}\right], \\ \frac{\log _{2}\left(N_{1}-1\right)}{N_{1}-2}\left(\Lambda-N_{1}\right)+\log _{2} N_{1}, & \Lambda \in\left[\frac{4\left(N_{1}-1\right)}{N_{1}}, N_{1}\right] .\end{cases}$

For isotropic states, this lower bound is exact.

It is direct to verify that the function $R(\Lambda)$ has only one reflection point for $N_{1}=2,3$. One can also easily verify this fact by plotting $R(\Lambda)$ for $N_{1}=4$. To show that the second derivative of $R$ with respect to $\Lambda$ has only one zero point for general $N_{1}$, for simplicity we replace $\log _{2}$ in (36) by the natural $\log$. Without confusion, the notion $R(\Lambda)$ below is still used, which, in fact, differs a positive factor $\log _{2} e$ from the $R(\Lambda)$ above.

First, it can be shown that there is one and only one point $\Lambda_{0}$ between 1 and $N_{1}-1$ such that $R^{\prime \prime}\left(\Lambda_{0}\right)=0$ for $N_{1} \geqslant 5$. The second derivative of $R$ with respect to $\Lambda$ is

$$
R^{\prime \prime}(\Lambda)=\gamma^{\prime \prime}(\Lambda) \log \frac{1-\gamma(\Lambda)}{\left(N_{1}-1\right) \gamma(\Lambda)}-\frac{1}{\Lambda\left(N_{1}-\Lambda\right)},
$$

where

$$
\gamma^{\prime \prime}(\Lambda)=-\frac{\sqrt{N_{1}-1}}{2}\left(\Lambda\left(N_{1}-\Lambda\right)\right)^{-3 / 2}
$$

Hence, $R^{\prime \prime}(1)=\lim _{\varepsilon \rightarrow 0} R^{\prime \prime}(1+\varepsilon)=+\infty$. On the other hand,

$$
R^{\prime \prime}\left(N_{1}-1\right)=-\frac{1}{N_{1}-1}\left(\log \frac{N_{1}-2}{2\left(N_{1}-1\right)}+1\right),
$$

which is less than 0 for $N_{1} \geqslant 5$. Therefore, for $N_{1} \geqslant 5$ there exists $\Lambda_{0} \in\left(1, N_{1}-1\right)$ such that $R^{\prime \prime}\left(\Lambda_{0}\right)=0$. From Eqs. (41) and (42) $\Lambda_{0}$ is the solution of $g(\Lambda)=f(\Lambda)$, where

$$
\begin{aligned}
& g(\Lambda)=\log \frac{1-\gamma(\Lambda)}{\left(N_{1}-1\right) \gamma(\Lambda)}, \\
& f(\Lambda)=-2 \sqrt{\frac{\Lambda\left(N_{1}-\Lambda\right)}{N_{1}-1}}
\end{aligned}
$$

As $g^{\prime}(\Lambda)>0, g(\Lambda)$ is a monotonically increasing function taking values from $g(1)=-\infty$ to 


$$
g\left(N_{1}-1\right)=2 \log \frac{N_{1}-2}{2\left(N_{1}-1\right)}>-2 .
$$

On the other hand, $f(1)=f\left(N_{1}-1\right)=-2, f^{\prime \prime}(\Lambda)>0$, i.e., $f$ is convex. Therefore, there is one and only one solution $\Lambda_{0}$ to the equation $g(\Lambda)=f(\Lambda)$ for $\Lambda \in\left(1, N_{1}-1\right)$.

Next, one can show that there are no solutions to $R^{\prime \prime}(\Lambda)=0$ for $\Lambda \in\left(N_{1}-1, N_{1}\right)$, i.e. $R^{\prime \prime}\left(N_{1}-1+\delta\right) \neq 0$, $\forall \delta \in(0,1)$. From (37), (41) and (42) this is equivalent to show $F(\delta) \equiv \frac{1}{2} B(\delta) \log A(\delta) \neq-1$, where,

$$
\begin{gathered}
B(\delta)=\sqrt{\frac{N_{1}-1}{\left(N_{1}-1+\delta\right)(1-\delta)}}, \\
A(\delta)=\frac{\left(N_{1} C(\delta)\right)^{2}-1}{N_{1}-1}, \\
C(\delta)=\left(\sqrt{N_{1}-1+\delta}+\sqrt{\left(N_{1}-1\right)(1-\delta)}\right)^{-1} .
\end{gathered}
$$

It is straightforward to verify that $A(0)>0$. As the derivative $C^{\prime}(\delta)$ of $C(\delta)$ with respect to $\delta$, is strictly positive, one has $A^{\prime}(\delta)>0$. Hence, $\log A(\delta)$ increases as $\delta$ increases. Similarly, as the derivative of $\left(N_{1}-1\right) /\left(\left(N_{1}-1+\delta\right)(1-\delta)\right)$ with respect to $\delta$ is positive, $B(\delta)$ also increases as $\delta$ increases. Therefore, $F(\delta)$ is an increasing function of $\delta$. Moreover, $\quad F(0)=\log \left(N_{1}-2\right) /\left(2\left(N_{1}-1\right)\right) \geqslant \log 3 / 8>-1$. It is seen that $F(\delta) \geqslant F(0)>-1, \forall \delta \in(0,1)$ and $N_{1} \geqslant 5$. Thus, $R^{\prime \prime}(\Lambda)=0$ has no solutions for $\Lambda \in\left(N_{1}-1, N_{1}\right)$ [38].

From the proof above, one has that both EOF (9) for isotropic states and the tight lower bound of EOF (40) are valid for arbitrary dimensions.

Another lower bound of EOF for bipartite states on even dimensional Hilbert spaces $N$ has been presented [29] from a new separability criterion [30]. On even dimensional spaces there exist antisymmetric unitary operations $V^{T}=-V$. The corresponding antiunitary maps $V(\cdot)^{T} V$, map any pure state to some state that is orthogonal to it. This leads to the conclusion that the map

$$
\Phi(\rho)=\operatorname{Tr}(\rho) I-\rho-V(\rho)^{T} V^{\dagger}
$$

is a positive but not completely positive map. It is nondecomposable. form:

The corresponding entanglement witness $W_{\Phi}$ has the

$$
W_{\Phi} \equiv N(I \otimes \Phi) P_{0},
$$

where the factor $N$ is introduced for convenience, $P_{0}$ represents the one-dimensional projection onto the maximally entangled singlet state. This criterion can detect some of the PPT entangled states. From this separability criterion a lower bound of EOF can be similarly obtained, $E(\rho) \geqslant$ $\operatorname{co}[R(\Lambda)]$, here $\Lambda \equiv \max \left\{\left\|\rho^{T_{1}}\right\|,\|\mathcal{R}(\rho)\|, 1-\operatorname{Tr}\left(W_{\Phi} \rho\right)\right\}$.

\subsection{Lower bounds of concurrence for bipartite mixed states}

The lower bound of the concurrence (7) for bipartite $2 \otimes N$ mixed states $\rho$ has been discussed in Refs. [2123]. Define the set of $N(N-1) / 2$ symmetric $2 N \times 2 N$ square matrices $S^{i j}, 1 \leqslant i \leqslant N-1, j>i$, to be the matrices whose elements $S_{m n}^{i j}$ are all zero except for

$$
S_{i, j+N}^{i j}=S_{j+N, i}^{i j}=1, \quad S_{j, i+N}^{i j}=S_{i+N, j}^{i j}=-1 .
$$

Then one has

$$
C(\rho) \geqslant \sqrt{\sum_{j>i} \sum_{i=1}^{N-1} C_{i j}^{2}(\rho)},
$$

where $C_{i j}(\rho)=\max \left(0, \lambda_{1}^{i j}-\sum_{k=2}^{4} \lambda_{k}^{i j}\right), \lambda_{l}^{i j}, l=1,2,3,4$ are the square roots, in decreasing order, of the four largest eigenvalues of the matrix $\sqrt{\rho} S^{i j} \rho^{*} S^{i j} \sqrt{\rho}$. This bound also gives rise to a corresponding lower bound for EOF,

$$
\varepsilon\left[\sqrt{\sum_{j>i} \sum_{i=1}^{N-1} C_{i j}^{2}(\rho)}\right],
$$

where $\varepsilon[x]$ is a monotonically increasing convex function of $x$ in its range $0 \leqslant x \leqslant 1$.

In fact for general bipartite states in $N_{1} \times N_{2}$, the squared concurrence has the form [47]:

$$
\begin{aligned}
C(|\psi\rangle)^{2} & =\sum_{m=1}^{D_{1}} \sum_{n=1}^{D_{2}}\left|C_{m n}\right|^{2} \\
& =4 \sum_{i<j}^{N_{1}} \sum_{k<l}^{N_{2}}\left|a_{i k} a_{j l}-a_{i l} a_{j k}\right|^{2},
\end{aligned}
$$

where $D_{1}=N_{1}\left(N_{1}-1\right) / 2, D_{2}=N_{2}\left(N_{2}-1\right) / 2, C_{m n}=\left\langle\psi \mid \tilde{\psi}_{m n}\right\rangle$, $\left|\tilde{\psi}_{m n}\right\rangle=\left(L_{m} \otimes L_{n}\right)\left|\psi^{*}\right\rangle$, and $L_{m}, m=1, \cdots, N_{1}\left(N_{1}-1\right) / 2, L_{n}$, $n=1, \cdots, N_{2}\left(N_{2}-1\right) / 2$ are the generators of group $S O$ $\left(N_{1}\right)$ and $S O\left(N_{2}\right)$, respectively.

From (46) it is evident that the $N_{1} \otimes N_{2}$ dimensional Hilbert space is decomposed into $N_{1}\left(N_{1}-1\right) N_{2}\left(N_{2}-1\right) / 4$ $2 \otimes 2$ dimensional subspaces, such that the squared concurrence is just the sum of all squared two-qubit's concurrences. A pure state is separable iff all these "two qubits" are separable.

Set $\left|\xi_{i}\right\rangle=\sqrt{p_{i}}\left|\psi_{i}\right\rangle$. The concurrence (7) takes the form:

$$
C(\rho)=\min \sum_{i}\left(\sum_{m=1}^{N_{1}\left(N_{1}-1\right) / 2} \sum_{n=1}^{N_{2}\left(N_{2}-1\right) / 2}\left|\left\langle\xi_{i}\left|L_{m} \otimes L_{n}\right| \xi_{i}^{*}\right\rangle\right|^{2}\right)^{\frac{1}{2}},
$$


by using the fact that any function $F=\sum_{i}\left(\sum_{j} x_{i j}^{2}\right)^{1 / 2}$ subjected to the constraints $z_{j}=\Sigma_{i} x_{i j}$ with $x_{i j}$ real and nonnegative, the inequality $\sum_{j} z_{j}^{2} \leqslant F^{2}$ holds and the procedure of extremization adopted in Refs. [10,25,26], one can prove that for an arbitrary $N_{1} \otimes N_{2}$ state (5), the concurrence $C(\rho)$ satisfies

$$
\tau(\rho) \equiv \sum_{m=1}^{N_{1}\left(N_{1}-1\right) / 2} \sum_{n=1}^{N_{2}\left(N_{2}-1\right) / 2} C_{m n}^{2} \leqslant C^{2}(\rho),
$$

where $\tau$ is the lower bound,

$$
C_{m n}=\max \left\{0, \lambda_{m n}^{(1)}-\lambda_{m n}^{(2)}-\lambda_{m n}^{(3)}-\lambda_{m n}^{(4)}\right\},
$$

$\lambda_{m n}^{(1)}, \cdots, \lambda_{m n}^{(4)}$ are the square roots of the four nonzero eigenvalues, in decreasing order, of the non-Hermitian matrix $\rho \tilde{\rho}_{m n}$, where $\tilde{\rho}_{m n}=\left(L_{m} \otimes L_{n}\right) \rho^{*}\left(L_{m} \otimes L_{n}\right)$.

The lower bound $\tau$ provides not only an effective separability criterion and an easy evaluation of entanglement, but also helps to classify mixed-state entanglement. It can be shown that a bipartite quantum state $\rho$ is distillable if $\tau\left(\rho^{\otimes M}\right)>0$ for some number $M$. For any pure tripartite state $|\phi\rangle_{123}$ in arbitrary $N_{1} \otimes N_{2} \otimes N_{3}$ dimensional spaces, the bound $\tau$ satisfies

$$
\tau\left(\rho_{12}\right)+\tau\left(\rho_{13}\right) \leqslant \tau\left(\rho_{1: 23}\right),
$$

where $\rho_{12}=\operatorname{Tr}_{3}\left(|\phi\rangle_{123}\langle\phi|\right), \rho_{13}=\operatorname{Tr}_{2}\left(|\phi\rangle_{123}\langle\phi|\right)$, and $\rho_{1: 23}=$ $\operatorname{Tr}_{23}\left(|\phi\rangle_{123}\langle\phi|\right)$.

Similar to the case of EOF, the separability criteria positive partial transpose (PPT) and realignment can also be used to obtain lower bounds of concurrence [27]. From (33) and (14) one has

$$
C(|\psi\rangle)=2 \sqrt{\sum_{i<j} \mu_{i} \mu_{j}}
$$

which varies smoothly from 0 for separable states to 2 $\left(N_{1}-1\right) / N_{1}$ for maximally entangled states.

By summing over all of the arithmetic mean inequalities $\mu_{i} \mu_{j}+\mu_{k} \mu_{l} \geqslant 2 \sqrt{\mu_{i} \mu_{j} \mu_{k} \mu_{l}}$ for $i<j$ and $k<l$, one gets

$$
\begin{aligned}
\sum_{i<j} \sum_{k<l}\left(\mu_{i} \mu_{j}+\mu_{k} \mu_{l}\right) & \geqslant 2 \sum_{i<j} \sum_{k<l} \sqrt{\mu_{i} \mu_{j} \mu_{k} \mu_{l}} \\
& =2\left(\sum_{i<j} \sqrt{\mu_{i} \mu_{j}}\right)^{2} .
\end{aligned}
$$

There are $N_{1}\left(N_{1}-1\right)$ terms of $\mu_{i} \mu_{j}$ on the left hand side of (52). Therefore

$$
\begin{aligned}
4 \sum_{i<j} \mu_{i} \mu_{j} & \geqslant \frac{2}{N_{1}\left(N_{1}-1\right)}\left(\left(\sum_{k} \sqrt{\mu_{k}}\right)^{2}-1\right)^{2} \\
& =\frac{8}{N_{1}\left(N_{1}-1\right)}\left(\sum_{i<j} \sqrt{\mu_{i} \mu_{j}}\right)^{2}
\end{aligned}
$$

From (53) one gets

$$
C\left(\rho^{i}\right) \geqslant \sqrt{2 /\left(N_{1}\left(N_{1}-1\right)\right)}\left(\left\|\left(\rho^{i}\right)^{T_{A}}\right\|-1\right),
$$

$$
C\left(\rho^{i}\right) \geqslant \sqrt{2 /\left(N_{1}\left(N_{1}-1\right)\right)}\left(\left\|\mathcal{R}\left(\rho^{i}\right)\right\|-1\right),
$$

for any pure state $\rho^{i}$, as from (35) one has

$$
\left\|\mathcal{R}\left(\rho^{i}\right)\right\|=\left\|\left(\rho^{i}\right)^{T_{A}}\right\|=\left(\sum_{k} \sqrt{\mu_{k}}\right)^{2},
$$

where $\sqrt{\mu_{k}}$ are the Schmidt coefficients for the pure state $\rho^{i}$.

Now assume $\Sigma_{i} p_{i} \rho^{i}$ is an optimal decomposition for $\rho$ to achieve the infimum of $C(\rho)$, where $\rho^{i}$ are pure state density matrices. Then $C(\rho)=\Sigma_{i} p_{i} C\left(\rho^{i}\right)$ by definition. Noticing that $\left\|\rho^{T_{A}}\right\| \leqslant \sum_{i} p_{i}\left\|\left(\rho^{i}\right)^{T_{A}}\right\|$ and $\|\mathcal{R}(\rho)\| \leqslant$ $\sum_{i} p_{i}\left\|\mathcal{R}\left(\rho^{i}\right)\right\|$ due to the convexity property of the trace norm, one can prove that for any $N_{1} \otimes N_{2}\left(N_{1} \leqslant N_{2}\right)$ mixed quantum state $\rho$, the concurrence $C(\rho)$ satisfies

$$
C(\rho) \geqslant \sqrt{\frac{2}{N_{1}\left(N_{1}-1\right)}}\left(\max \left(\left\|\rho^{T_{1}}\right\|,\|\mathcal{R}(\rho)\|\right)-1\right) .
$$

For the $U \otimes U^{*}$ invariant mixed isotropic states with $N_{1}=N_{2}=N[39,40]$, the bound (54) gives the exact value of the concurrence derived in Ref. [20].

If one takes the separability criterion (44) into account, the above bound can be improved [29]. Set $f_{\mathrm{ppt}}(\rho)=$ $\left\|\rho^{T_{1}}\right\|-1, \quad f_{\text {realign }}(\rho)=\|\mathcal{R}(\rho)\|-1, \quad f_{W_{\Phi}}(\rho)=-\operatorname{Tr}\left(W_{\Phi} \rho\right)$, where $W_{\Phi}$ is defined in Eq. (44). Then

$$
C(\rho) \geqslant \sqrt{\frac{2}{N_{1}\left(N_{1}-1\right)}} \max \left(f_{\mathrm{ppt}}(\rho), f_{\text {realign }}(\rho), f_{W_{\Phi}}(\rho)\right),
$$

holds for any $N_{1} \otimes N_{2}\left(N_{1} \leqslant N_{2}\right)$ mixed quantum state $\rho$.

An interesting separability criterion called local uncertainty relations (LURs) criterion is based on uncertainty relations [50]. It can detect some of the PPT entangled states [51,52]. It says that if $\left\{A_{i}\right\}$ and $\left\{B_{i}\right\}$ are observables acting on $\mathcal{H}_{1}$ and $\mathcal{H}_{2}$ respectively, fulfilling the uncertainty relations $\sum_{i} \Delta_{\rho}^{2}\left(A_{i}\right) \geqslant C_{A}$ and $\sum_{i} \Delta_{\rho}^{2}\left(B_{i}\right) \geqslant C_{B}\left(C_{A}, C_{B} \geqslant 0\right)$, then,

$$
\sum_{i} \Delta_{\rho}^{2}\left(A_{i} \otimes I+I \otimes B_{i}\right) \geqslant C_{A}+C_{B}
$$

holds for separable states [50]. The variance $\Delta^{2}$ is given by $\Delta_{\rho}^{2}(M)=\left\langle M^{2}\right\rangle_{\rho}-\langle M\rangle_{\rho}^{2}$, where $\langle M\rangle_{\rho}=\operatorname{Tr}(\rho M)$ is the expectation value of the observable $M$. A particularly interesting choice of the observables is the local orthogonal 
observables (LOOs) [53], that is, the orthonormal bases of $\mathcal{B}\left(\mathcal{H}_{1}\right)$ and $\mathcal{B}\left(\mathcal{H}_{2}\right)$, denoted by $\left\{G_{i}^{A}\right\}_{i=1}^{N_{1}^{2}}$ and $\left\{G_{i}^{B}\right\}_{i=1}^{N_{2}^{2}}$. Since

$$
\sum_{i=1}^{N_{1}^{2}} \Delta_{\rho}^{2}\left(G_{i}^{A}\right) \geqslant N_{1}-1, \quad \sum_{i=1}^{N_{2}^{2}} \Delta_{\rho}^{2}\left(G_{i}^{B}\right) \geqslant N_{2}-1,
$$

in this case (55) reads [52]

$$
\sum_{i=1}^{N_{2}^{2}} \Delta_{\rho}^{2}\left(G_{i}^{A} \otimes I+I \otimes G_{i}^{B}\right) \geqslant N_{1}+N_{2}-2 .
$$

For any set of LOOs $\left\{G_{i}^{A}\right\}$ and $\left\{G_{i}^{B}\right\}$ and any $N_{1} \times N_{2}$ $\left(N_{1} \leqslant N_{2}\right)$ pure state $|\psi\rangle$ with Schmidt decomposition (33), using (56), one can obtain that

$$
\begin{aligned}
& \sum_{i} \Delta_{\psi}^{2}\left(G_{i}^{A} \otimes I+I \otimes G_{i}^{B}\right) \geqslant N_{1}+N_{2}-2 \\
& \quad+2 \sum_{i}\left(\left\langle G_{i}^{A} \otimes G_{i}^{B}\right\rangle_{\psi}-\left\langle G_{i}^{A}\right\rangle_{\rho_{A}}\left\langle G_{i}^{B}\right\rangle_{\rho_{B}}\right) .
\end{aligned}
$$

Furthermore, due to the fact that

$$
\sum_{i}\left(\left\langle G_{i}^{A} \otimes G_{i}^{B}\right\rangle_{\psi}-\left\langle G_{i}^{A}\right\rangle_{\rho_{A}}\left\langle G_{i}^{B}\right\rangle_{\rho_{B}}\right) \geqslant-2 \sum_{j<k} \sqrt{\mu_{j} \mu_{k}},
$$

one has

$$
\sum_{i=1}^{N_{2}^{2}} \Delta_{\psi}^{2}\left(G_{i}^{A} \otimes I+I \otimes G_{i}^{B}\right) \geqslant N_{1}+N_{2}-2-4 \sum_{j<k} \sqrt{\mu_{j} \mu_{k}} .(58)
$$

Let $\Sigma_{n} p_{n}\left|\psi_{n}\right\rangle\left\langle\psi_{n}\right|$ be the decomposition of $\rho$ for which the minimum in (7) is attained, so that, $C(\rho)=\Sigma_{n} p_{n} C\left(\psi_{n}\right)$. From (51) and (58), a lower bound of concurrence based on LURs criterion is obtained [31]: For any $N_{1} \times N_{2}$ $\left(N_{1} \leqslant N_{2}\right)$ quantum state $\rho$,

$$
C(\rho) \geqslant \frac{N_{1}+N_{2}-2-\sum_{i} \Delta_{\rho}^{2}\left(G_{i}^{A} \otimes I+I \otimes G_{i}^{B}\right)}{\sqrt{2 N_{1}\left(N_{1}-1\right)}}
$$

for any set of LOOs $\left\{G_{i}^{A}\right\}$ and $\left\{G_{i}^{B}\right\}$.

The bound (59) depends on the choice of the local orthonormal observables. In Ref. [32], this bound is optimized. For a given state $\rho$, one can choose an arbitrary complete set of LOOs $\left\{G_{k}^{A}\right\},\left\{G_{k}^{B}\right\}$. The other orthonormal normalized basis of the local orthonormal observable space can be obtained from $\left\{G_{k}^{A}\right\}$ and $\left\{G_{k}^{B}\right\}$ by unitary transformations $U$ and $V$ :

$$
\widetilde{G_{k}^{A}}=\sum_{l} U_{k l} G_{l}^{A}, \widetilde{G_{k}^{B}}=\sum_{m} V_{k m} G_{m}^{B} .
$$

Select $U$ and $V$ so that $\tau=U^{\dagger} \Lambda V$ is the singular value decomposition of thematrix $\tau$ defined by $\tau_{l m}=\left\langle G_{l}^{A} \otimes G_{m}^{B}\right\rangle-\left\langle G_{l}^{A} \otimes I\right\rangle$ $\left\langle I \otimes G_{m}^{B}\right\rangle$. Then the new observables can be written as

$$
\widetilde{G_{k}^{A}}=\sum_{l} U_{i l}\left\{G_{l}^{A}\right\}, \widetilde{G_{k}^{B}}=-\sum_{m} V_{j m}^{*}\left\{G_{m}^{A}\right\} .
$$

One has

$$
\begin{aligned}
& \sum_{k} \Delta_{\rho}^{2}\left(\widetilde{G_{k}^{A}} \otimes I+I \otimes \widetilde{G_{k}^{B}}\right) \\
& \quad=N_{1}-\operatorname{Tr} \rho_{A}^{2}+N_{2}+\operatorname{Tr} \rho_{B}^{2}-2 \sum_{k} \sigma_{k}(\tau),
\end{aligned}
$$

where $\sigma_{k}(\tau)$ stands for the $k$ th singular value of $\tau$.

Since the entanglement criterion based on local uncertainty relations is strictly stronger than the realignment criterion [52], one has the following inequality [32]:

$$
C(\rho) \geqslant \sqrt{\frac{2}{N_{1}\left(N_{1}-1\right)}}\left(\max \left(\left\|\rho^{T_{A}}\right\|, \mathcal{L}_{\max }(\rho)\right)-1\right),
$$

for any $N_{1} \otimes N_{2}\left(N_{1} \leqslant N_{2}\right)$ mixed quantum state $\rho$, where $\mathcal{L}_{\max }=\sum_{k} \sigma_{k}(\tau)+\left(\operatorname{Tr} \rho_{A}^{2}+\operatorname{Tr} \rho_{B}^{2}\right) / 2$.

In Ref. [54], a separability criterion based on the Bloch representation of density matrices has been presented. This correlation matrix criterion says that for bipartite separable states $\rho$,

$$
\|T\| \leqslant \sqrt{\frac{N_{1} N_{2}\left(N_{1}-1\right)\left(N_{2}-1\right)}{4}},
$$

where $T$ is an $\left(N_{1}^{2}-1\right) \times\left(N_{2}^{2}-1\right)$ matrix with $T_{i j}=N_{1} N_{2} / 4$. $\operatorname{Tr}\left(\rho \lambda_{i}^{A} \otimes \lambda_{j}^{B}\right), \lambda_{k}^{A}$ and $\lambda_{k}^{B}$ are the generators of $S U\left(N_{1}\right)$ and $S U\left(N_{2}\right)$ respectively, satisfying $\operatorname{Tr} \lambda_{k}^{(A / B)} \lambda_{l}^{(A / B)}=2 \delta_{k l}$.

Based on the correlation matrix criterion a lower bound of concurrence is obtained in Ref. [31]. For any $N_{1} \times N_{2}$ $\left(N_{1} \leqslant N_{2}\right)$ quantum state $\rho$, one has

$$
C(\rho) \geqslant \sqrt{\frac{8}{N_{1}^{3} N_{2}^{2}\left(N_{1}-1\right)}}\left(\|T\|-K_{N_{1} N_{2}}\right),
$$

where $K_{N_{1} N_{2}}=\sqrt{N_{1} N_{2}\left(N_{1}-1\right)\left(N_{2}-1\right)} / 2$.

Example 1 Consider the $3 \times 3$ bound entangled state [55],

$$
\rho=\frac{1}{4}\left(I_{9}-\sum_{i=0}^{4}\left|\xi_{i}\right\rangle\left\langle\xi_{i}\right|\right),
$$

where $I_{9}$ is the $9 \times 9$ identity matrix,

$$
\begin{aligned}
& \left|\xi_{0}\right\rangle=\frac{1}{\sqrt{2}}|0\rangle(|0\rangle-|1\rangle), \\
& \left|\xi_{1}\right\rangle=\frac{1}{\sqrt{2}}(|0\rangle-|1\rangle)|2\rangle, \\
& \left|\xi_{2}\right\rangle=\frac{1}{\sqrt{2}}|2\rangle(|1\rangle-|2\rangle), \\
& \left|\xi_{3}\right\rangle=\frac{1}{\sqrt{2}}(|1\rangle-|2\rangle)|0\rangle, \\
& \left|\xi_{4}\right\rangle=\frac{1}{3}(|0\rangle+|1\rangle+|2\rangle)(|0\rangle+|1\rangle+|2\rangle) .
\end{aligned}
$$

Choose the local orthonormal observables to be the normalized generators of $S U(3)$. (63) gives $C(\rho) \geqslant 0.0205$. (54) gives $C(\rho) \geqslant 0.050$. (59) gives $C(\rho) \geqslant 0.052$ [31], while (61) yields a better lower bound $C(\rho) \geqslant 0.055$. 
Example 2 Consider the $4 \times 4$ bound entangled states [56],

$$
\rho=\left[\begin{array}{cccccccccccccccc}
\frac{1-\varepsilon}{4} & 0 & 0 & 0 & 0 & 0 & 0 & 0 & 0 & 0 & 0 & 0 & 0 & 0 & 0 & 0 \\
0 & \frac{\varepsilon}{8} & 0 & 0 & -\frac{\varepsilon}{8} & 0 & 0 & 0 & 0 & 0 & 0 & 0 & 0 & 0 & 0 & 0 \\
0 & 0 & \frac{\varepsilon}{8} & 0 & 0 & 0 & 0 & 0 & -\frac{\varepsilon}{8} & 0 & 0 & 0 & 0 & 0 & 0 & 0 \\
0 & 0 & 0 & 0 & 0 & 0 & 0 & 0 & 0 & 0 & 0 & 0 & 0 & 0 & 0 & 0 \\
0 & -\frac{\varepsilon}{8} & 0 & 0 & \frac{\varepsilon}{8} & 0 & 0 & 0 & 0 & 0 & 0 & 0 & 0 & 0 & 0 & 0 \\
0 & 0 & 0 & 0 & 0 & \frac{1-\varepsilon}{4} & 0 & 0 & 0 & 0 & 0 & 0 & 0 & 0 & 0 & 0 \\
0 & 0 & 0 & 0 & 0 & 0 & 0 & 0 & 0 & 0 & 0 & 0 & 0 & 0 & 0 & 0 \\
0 & 0 & 0 & 0 & 0 & 0 & 0 & \frac{\varepsilon}{8} & 0 & 0 & 0 & 0 & 0 & -\frac{\varepsilon}{8} & 0 & 0 \\
0 & 0 & -\frac{\varepsilon}{8} & 0 & 0 & 0 & 0 & 0 & \overline{8} & 0 & 0 & 0 & 0 & 0 & 0 & 0 \\
0 & 0 & 0 & 0 & 0 & 0 & 0 & 0 & 0 & 0 & 0 & 0 & 0 & 0 & 0 & 0 \\
0 & 0 & 0 & 0 & 0 & 0 & 0 & 0 & 0 & 0 & \frac{1-\varepsilon}{4} & 0 & 0 & 0 & 0 & 0 \\
0 & 0 & 0 & 0 & 0 & 0 & 0 & 0 & 0 & 0 & 0 & \frac{\varepsilon}{8} & 0 & 0 & -\frac{\varepsilon}{8} & 0 \\
0 & 0 & 0 & 0 & 0 & 0 & 0 & 0 & 0 & 0 & 0 & 0 & 0 & 0 & 0 & 0 \\
0 & 0 & 0 & 0 & 0 & 0 & 0 & -\frac{\varepsilon}{8} & 0 & 0 & 0 & 0 & 0 & \frac{\varepsilon}{8} & 0 & 0 \\
0 & 0 & 0 & 0 & 0 & 0 & 0 & 0 & 0 & 0 & 0 & -\frac{\varepsilon}{8} & 0 & 0 & \frac{\varepsilon}{8} & 0 \\
0 & 0 & 0 & 0 & 0 & 0 & 0 & 0 & 0 & 0 & 0 & 0 & 0 & 0 & 0 & \frac{1-\varepsilon}{4}
\end{array}\right],
$$

where $0 \leqslant \varepsilon \leqslant \frac{1}{2}$. Equations (54), (61), (63) give the same result: $C(\rho) \geqslant 0$. This result shows that the realignment, LURs and correlation matrix criteria fail to detect this bound entangled state.

\subsection{Lower bounds of concurrence for tripartite systems}

Let $\mathcal{H}_{1}, \mathcal{H}_{2}, \cdots, \mathcal{H}_{M}$ be $M(\geqslant 2) N_{1}, N_{2}, \ldots, N_{M}$-dimensional Hilbert spaces respectively. The concurrence for a general pure multipartite state $|\psi\rangle \in \mathcal{H}_{1} \otimes \mathcal{H}_{2} \otimes \cdots \otimes \mathcal{H}_{M}$ is defined by

$$
C(|\psi\rangle)=\sqrt{m-\sum_{\alpha=1}^{m} \operatorname{Tr} \rho_{\alpha}^{2}}
$$

where $m=2^{M-1}-1$ is the number of all possible bipartite separations of an $M$-partite system, the reduced density matrix $\rho_{\alpha}, \alpha=1, \ldots, m$, is obtained by tracing over one part of the subsystems associated with the $\alpha$-th bipartite separation.

For the multipartite case, a Schmidt expression like (33) does not exist. To get a lower bound of the multipartite concurrence, one needs the operations of generalized partial transpose and realignment. Let us first recall some notations used in various matrix operations [57,58]. A generic matrix $G$ can always be written as $G=\sum_{i, j} a_{i j}\langle j|\otimes| i\rangle$, where $|i\rangle,|j\rangle$ are vectors of a suitably selected normalized real orthogonal basis. The operations $\mathcal{T}_{r}\left(\operatorname{resp} . \mathcal{T}_{c}\right)$ are defined to be the row transposition (resp. column transposition) of $G$ which transposes the second (resp. first) vector in the above tensor product expression of $G$ :

$$
\begin{aligned}
& \mathcal{T}_{r}(G)=\sum_{i, j} a_{i j}\langle j| \otimes\langle i|, \\
& \mathcal{T}_{c}(G)=\sum_{i, j} a_{i j}|j\rangle \otimes|i\rangle .
\end{aligned}
$$

It is easily verified that $\mathcal{T}_{c} \mathcal{T}_{r}(G)=\mathcal{T}_{r} \mathcal{T}_{c}(G)=G^{T}$, where $T$ denotes matrix transposition.

In the following, $\mathcal{T}_{r_{k}}\left(\operatorname{resp} . \mathcal{T}_{c_{k}}\right.$ ) are defined to be the row (resp. column) transpositions with respect to the subsystem $k$. For instance, $\mathcal{T}_{r_{12}}$ stands for the row transpositions with respect to the subsystems 1 and 2 . Let $\mathcal{Y}=\left\{x_{1}, x_{2}, \ldots\right\}$ be a set of such operations on a density matrix $\quad \rho$. Set $\quad \rho^{\mathcal{T}_{\mathcal{Y}}}=\mathcal{T}_{\mathcal{Y}}(\rho)=\mathcal{T}_{x_{1}} \mathcal{T}_{x_{2}} \ldots(\rho)$, e.g. $\rho^{\mathcal{T}\left\{c_{1}, r_{2}, r_{3}\right\}} \equiv \mathcal{T}_{\left\{c_{1}\right\}} \mathcal{T}_{\left\{r_{2}\right\}} \mathcal{T}_{\left\{r_{3}\right\}}(\rho)$.

The concurrence for a general pure tripartite state $|\psi\rangle \in \mathcal{H}_{1} \otimes \mathcal{H}_{2} \otimes \mathcal{H}_{3}$ is defined by

$$
C(|\psi\rangle)=\sqrt{3-\operatorname{Tr}\left(\rho_{1}^{2}+\rho_{2}^{2}+\rho_{3}^{2}\right)},
$$


where the reduced density matrix $\rho_{1}$ (resp. $\rho_{2}, \rho_{3}$ ) is obtained by tracing over the subsystems 2 and 3 (resp. 1 and 3, 1 and 2). In Ref. [33] a special class of $\mathcal{Y}$ is discussed: $\mathcal{Y}_{i}=\left\{c_{i}, r_{i}\right\}, i=1,2,3, \quad \mathcal{Y}_{4}=\left\{c_{1}, r_{23}\right\}, \quad \mathcal{Y}_{5}=\left\{c_{12}, r_{3}\right\}$, $\mathcal{Y}_{6}=\left\{c_{13}, r_{2}\right\}$. As $\rho^{\mathcal{T} y_{i}}=\rho^{T_{i}}, i=1,2,3$, where $T_{i}$ stands for the partial transposition with respect to the subsystem $i$, the operations $\mathcal{Y}_{1}, \mathcal{Y}_{2}$ and $\mathcal{Y}_{3}$ correspond to the partial transpositions of $\rho$.

For the most simple tripartite system, the three qubits case, a state $|\Psi\rangle$ can be written in terms of the generalized Schmidt decomposition [59],

$$
\begin{aligned}
|\Psi\rangle= & \lambda_{0}|000\rangle+\lambda_{1} e^{i \phi}|100\rangle+\lambda_{2}|101\rangle \\
& +\lambda_{3}|110\rangle+\lambda_{4}|111\rangle
\end{aligned}
$$

with normalization condition $\lambda_{i} \geqslant 0,0 \leqslant \phi \leqslant \pi, \sum_{i} \lambda_{i}^{2}=1$. The corresponding density matrix $\rho=|\Psi\rangle\langle\Psi|$ has the following properties:

$$
\begin{aligned}
& \operatorname{Tr} \rho_{1}^{2}=1-2 \mu_{0}\left(1-\mu_{0}-\mu_{1}\right), \\
& \operatorname{Tr} \rho_{2}^{2}=1-2 \mu_{0}\left(1-\mu_{0}-\mu_{1}-\mu_{2}\right)-2 \Delta, \\
& \operatorname{Tr} \rho_{3}^{2}=1-2 \mu_{0}\left(1-\mu_{0}-\mu_{1}-\mu_{3}\right)-2 \Delta,
\end{aligned}
$$

where $\Delta \equiv\left|\lambda_{1} \lambda_{4} e^{i \phi}-\lambda_{2} \lambda_{3}\right|^{2}, \quad \mu_{i}=\lambda_{i}^{2}, \quad i=0, \quad 1, \quad \ldots, \quad 4$ Therefore, from (68) one has

$$
C^{2}(\rho)=2 \mu_{0}\left(3-3 \mu_{0}-3 \mu_{1}-\mu_{2}-\mu_{3}\right)+4 \Delta,
$$

which varies smoothly from 0 , for pure product states, to $3 / 2$ for maximally entangled pure states.

On the other hand, under the operations of $\mathcal{Y}_{i}$, $i=1,2,3$, one gets

$$
\begin{aligned}
& \left\|\rho^{\mathcal{T}_{y_{1}}}\right\|=1+2 \sqrt{\mu_{0}\left(\mu_{2}+\mu_{3}+\mu_{4}\right)}, \\
& \left\|\rho^{\mathcal{T}_{y_{2}}}\right\|=1+2 \sqrt{\Delta+\mu_{0}\left(\mu_{3}+\mu_{4}\right)}, \\
& \left\|\rho^{\mathcal{T}_{y_{3}}}\right\|=1+2 \sqrt{\Delta+\mu_{0}\left(\mu_{2}+\mu_{4}\right)} .
\end{aligned}
$$

Combining (70) and (71) one can obtain

$$
C(\rho) \geqslant\left(\left\|\rho^{\mathcal{T}_{\mathcal{Y}_{j}}}\right\|-1\right), \quad j=1,2,3 .
$$

A three-qubit $(2 \otimes 2 \otimes 2)$ system can be viewed as three different bipartite $(2 \otimes 4$ or $4 \otimes 2)$ systems. From the results for bipartite systems in Section 3.2, these three bipartite separations give rise to, respectively

$$
\begin{aligned}
& 1-\operatorname{Tr}\left(\rho_{1}^{2}\right) \geqslant \frac{1}{2}\left(\left\|\rho^{\mathcal{T}\left\{c_{1}, r_{23}\right\}}\right\|-1\right)^{2}, \\
& 1-\operatorname{Tr}\left(\rho_{2}^{2}\right) \geqslant \frac{1}{2}\left(\left\|\rho^{\mathcal{T}\left\{c_{13}, r_{2}\right\}}\right\|-1\right)^{2}, \\
& 1-\operatorname{Tr}\left(\rho_{3}^{2}\right) \geqslant \frac{1}{2}\left(\left\|\rho^{\mathcal{T}}\left\{c_{12}, r_{3}\right\}\right\|-1\right)^{2} .
\end{aligned}
$$

Therefore,

$$
C(\rho) \geqslant \frac{1}{\sqrt{2}} \max \left\{\left\|\rho^{\mathcal{T}_{y_{j}}}\right\|-1\right\}, \quad j=4,5,6 .
$$

Hence, if one assumes that $\Sigma_{i} p_{i} \rho^{i}$ is the optimal decomposition of $\rho$ such that $C(\rho)=\Sigma_{i} p_{i} C\left(\rho^{i}\right)$, where $\rho^{i}$ are pure state density matrices, taking into account that $\left\|\rho^{\mathcal{T}_{\mathcal{Y}}}\right\| \leqslant \sum_{i} p_{i}\left\|\left(\rho^{i}\right)^{\mathcal{T}_{\mathcal{Y}}}\right\|$, from (72) and (73) one gets that for any three-qubit mixed quantum state $\rho$, the concurrence $C(\rho)$ satisfies

$$
C(\rho) \geqslant \max \left\{\left\|\rho^{\mathcal{T}_{\mathcal{Y}_{i}}}\right\|-1, \frac{1}{\sqrt{2}}\left(\left\|\rho^{\mathcal{T}_{\mathcal{Y}_{j}}}\right\|-1\right)\right\},
$$

where $i=1,2,3 ; j=4,5,6$.

For higher dimensional tripartite systems, an expression like (69) does not exist. The related lower bound of concurrence will be discussed in the next section for arbitrary multipartite systems.

\subsection{Lower bounds of concurrence for multipartite systems}

\subsubsection{Generalized Greenberger-Horne-Zeilinger state}

. Concerning multipartite $(M>3)$ systems, let us first consider the $M$-partite generalized Greenberger-HorneZeilinger (GHZ) state,

$$
|\Phi\rangle=\cos \theta|00 \cdots 0\rangle+\sin \theta|11 \cdots 1\rangle .
$$

For $\rho=|\Phi\rangle\langle\Phi|$, one gets $\rho_{i}=\operatorname{Tr}_{\{1, \cdots, i-1, i+1, \cdots, M\}} \rho=$ $\cos ^{2} \theta|0\rangle\left\langle 0\left|+\sin ^{2} \theta\right| 1\right\rangle\langle 1| . \quad$ Therefore, $\quad \operatorname{Tr} \rho_{i}^{2}=\cos ^{4} \theta+$ $\sin ^{4} \theta=1-2 \sin ^{2} \theta \cos ^{2} \theta, i=1,2, \ldots, M$. In fact, one can prove that $\operatorname{Tr} \rho_{i_{1} i_{2} \ldots i_{m}}^{2}=1-2 \sin ^{2} \theta \cos ^{2} \theta$ for all $i_{1} \neq i_{2} \neq \cdots \neq i_{m} \in\{1,2, \ldots, M\}, 1 \leqslant m \leqslant M$. Hence, from (66) one has

$$
C(\rho)=\sqrt{2 d \sin ^{2} \theta \cos ^{2} \theta} .
$$

On the other hand, the partial transpose of $\rho$ with respect to the $i$ th qubit space gives rise to

$$
\begin{aligned}
\rho^{T_{i}}= & \cos ^{2} \theta\left|0 \cdots 0_{i} \cdots 0\right\rangle\left\langle 0 \cdots 0_{i} \cdots 0\right| \\
& +\cos \theta \sin \theta\left|0 \cdots 1_{i} \cdots 0\right\rangle\left\langle 1 \cdots 0_{i} \cdots 1\right| \\
& +\cos \theta \sin \theta\left|1 \cdots 0_{i} \cdots 1\right\rangle\left\langle 0 \cdots 1_{i} \cdots 0\right| \\
& +\sin ^{2} \theta\left|1 \cdots 1_{i} \cdots 1\right\rangle\left\langle 1 \cdots 1_{i} \cdots 1\right|,
\end{aligned}
$$

$i=1,2, \cdots, M$. As $\rho^{T_{i}}$ is Hermitian, its singular values are simply given by the square root of the eigenvalues of $\left(\rho^{T_{i}}\right)^{2}$. The trace norm of $\rho^{T_{i}}$ takes the form $\left\|\rho^{T_{i}}\right\|=$ $1+2 \sqrt{\sin ^{2} \theta \cos ^{2} \theta}$. The trace norms of partial transposed $\rho$ with respect to the other sub-qubit spaces can be similarly calculated. All together one gets 


$$
\left\|\rho^{T_{i_{1} i_{2} \cdots i_{m}}}\right\|=1+2 \sqrt{\sin ^{2} \theta \cos ^{2} \theta},
$$

where $i_{1} \neq i_{2} \neq \cdots \neq i_{m} \in\{1,2, \ldots, M\}, 1 \leqslant m \leqslant M$.

Now consider the norm of $\rho$ under bipartite realignment. If a bipartite realignment with respect to the subsystems $i$ and $j, 1 \leqslant i \neq j \leqslant M$ is made, while leaving the other subsystems untouched, one has

$$
\begin{aligned}
& \mathcal{R}_{i \mid j}(\rho) \\
& =\cos ^{2} \theta\left|0 \cdots 0_{i} \cdots 0_{j} \cdots 0\right\rangle\left\langle 0 \cdots 0_{i} \cdots 0_{j} \cdots 0\right| \\
& \quad+\cos \theta \sin \theta\left|0 \cdots 0_{i} \cdots 1_{j} \cdots 0\right\rangle\left\langle 1 \cdots 0_{i} \cdots 1_{j} \cdots 1\right| \\
& \quad+\cos \theta \sin \theta\left|1 \cdots 1_{i} \cdots 0_{j} \cdots 1\right\rangle\left\langle 0 \cdots 1_{i} \cdots 0_{j} \cdots 0\right| \\
& \quad+\sin ^{2} \theta\left|1 \cdots 1_{i} \cdots 1_{j} \cdots 1\right\rangle\left\langle 1 \cdots 1_{i} \cdots 1_{j} \cdots 1\right| .
\end{aligned}
$$

Therefore, $\left\|\mathcal{R}_{i \mid j}(\rho)\right\|=1+2 \sqrt{\sin ^{2} \theta \cos ^{2} \theta}$. Let $\Theta_{1}$ and $\Theta_{2}$ be two different subsystems. One can similarly verify that

$$
\left\|\mathcal{R}_{\Theta_{1} \mid \Theta_{2}}(\rho)\right\|=1+2 \sqrt{\sin ^{2} \theta \cos ^{2} \theta} .
$$

From (76), (77) and (78) one can prove that for any $M$ qubit mixed state with decomposition $\rho=\Sigma_{i} p_{i}\left|\Psi_{i}\right\rangle\left\langle\Psi_{i}\right|$, if $\left|\Psi_{i}\right\rangle$ can be written in the form (75) for all $i$, then the concurrence $C(\rho)$ satisfies

$$
C(\rho) \geqslant \max \left\{\left\|\rho^{T_{\Theta}}\right\|,\left\|\mathcal{R}_{\Theta_{1} \mid \Theta_{2}}(\rho)\right\|\right\}-1,
$$

where $\Theta, \Theta_{1}, \Theta_{2}$ are subsets of the indices $\{1,2, \ldots, M\}$, such that $\Theta_{1} \cup \Theta_{2}=\varnothing$.

Remark Once a density matrix has a decomposition with all the pure states of the form (75), then all other possible decompositions of it will also have the form (75), since other decompositions can be obtained from the unitarily linear combinations of this decomposition, and any linear combinations of the type (75) still have the form (75).

\subsubsection{Generalized $W$-state}

We consider now another M-qubit state, the generalized $W$-state,

$$
\begin{aligned}
|\Psi\rangle= & a_{1}|10 \cdots 0\rangle+a_{2}|01 \cdots 0\rangle+\cdots \\
& +a_{M}|00 \cdots 1\rangle .
\end{aligned}
$$

Let $\rho=|\Psi\rangle\langle\Psi|$, then

$$
\rho_{i}=\operatorname{Tr}_{\{1, \cdots, i-1, i+1, \cdots, M\}} \rho=\left|a_{i}\right|^{2}|1\rangle\left\langle 1\left|+\left(\sum_{j \neq i}\left|a_{j}\right|^{2}\right)\right| 0\right\rangle\langle 0| .
$$

Therefore, $\quad \operatorname{Tr} \rho_{i}^{2}=\left|a_{i}\right|^{4}+\left(\sum_{j \neq i}\left|a_{j}\right|^{2}\right)^{2}, i=1,2, \ldots, M$.

Generally, one can prove that

$$
\begin{aligned}
\operatorname{Tr} \rho_{i_{1} i_{2} \cdots i_{m}}^{2}= & \left(\left|a_{i_{1}}\right|^{2}+\left.a_{i_{2}}\right|^{2}+\cdots+\left.a_{i_{m}}\right|^{2}\right)^{2} \\
& +\left(\sum_{k \neq\left\{i_{1}, i_{2}, \cdots, i_{m}\right\}}\left|a_{k}\right|^{2}\right)^{2}
\end{aligned}
$$

for all $i_{1} \neq i_{2} \neq \cdots \neq i_{m} \in\{1,2, \ldots, M\}, 1 \leqslant m \leqslant M$. Hence from (66),

$$
C(\rho)=\sqrt{2^{M-1} \sum_{i<j}\left|a_{i} a_{j}\right|^{2}} .
$$

From a direct calculation, the trace norm of the partial transposed matrix $\rho^{T_{i}}$ of $\rho$ with respect to the $i$ th qubit space is given by $\left\|\rho^{T_{i}}\right\|=1+2 \sqrt{\sum_{j \neq i}\left|a_{i} a_{j}\right|^{2}}$. The trace norms of the partial transposed $\rho$ with respect to the other sub-qubit spaces can also be similarly calculated,

$$
\left\|\rho^{T_{i_{1} i_{2} \cdots i_{m}}}\right\|=1+2 \sqrt{\sum_{l \neq\left\{i_{1}, i_{2}, \cdots, i_{m}\right\}} \sum_{k=i_{1}}^{i_{m}}\left|a_{k} a_{l}\right|^{2},}
$$

where $i_{1} \neq i_{2} \neq \cdots \neq i_{m} \in\{1,2, \ldots, M\}, 1 \leqslant m \leqslant M$.

An $M$-qubit $W$ state can be viewed as $m$ different bipartite systems. Let $\Gamma_{\alpha}^{1}, \Gamma_{\alpha}^{2}$ denote two subsets of the indices $\quad\{1, \quad 2, \quad \ldots, \quad M\}, \quad \Gamma_{\alpha}^{1} \cap \Gamma_{\alpha}^{2}=\varnothing, \Gamma_{\alpha}^{1} \cup \Gamma_{\alpha}^{2}=$ $\{1,2, \ldots, M\}, \alpha=1, \cdots, m$. From the results for bipartite systems, these $m$ bipartite separations give rise to, respectively,

$$
\begin{aligned}
& 1-\operatorname{Tr}\left(\rho_{\Gamma_{\alpha}^{1}}^{2}\right) \geqslant \frac{1}{2}\left(\left\|\mathcal{R}_{\Gamma_{\alpha}^{1} \mid \Gamma_{\alpha}^{2}}(\rho)\right\|-1\right)^{2}, \\
& \alpha=1, \cdots, m .
\end{aligned}
$$

Hence,

$$
\begin{aligned}
C(\rho) & =\sqrt{m-\sum_{\alpha=1}^{m} \operatorname{Tr}\left(\rho_{\Gamma_{\alpha}^{1}}^{2}\right)} \\
& \geqslant \frac{1}{\sqrt{2}} \max \left\{\left\|\mathcal{R}_{\Gamma_{\alpha}^{1} \mid \Gamma_{\alpha}^{2}}(\rho)\right\|-1, \quad \alpha=1, \cdots, m\right\} .
\end{aligned}
$$

Therefore, for any $M$-qubit mixed state with decomposition with respect to the generalized $W$ states, $\rho=\Sigma_{i} p_{i}\left|\Psi_{i}\right\rangle\left\langle\Psi_{i}\right|$, such that $\left|\Psi_{i}\right\rangle$ can be written in the form (80) for all $i$, the concurrence $C(\rho)$ satisfies

$$
\begin{aligned}
& C(\rho) \geqslant \\
& \max \left\{\left\|\rho^{T_{\Gamma_{\alpha}^{1}}}\right\|-1, \frac{1}{\sqrt{2}}\left(\left\|\mathcal{R}_{\Gamma_{\alpha}^{1} \mid \Gamma_{\alpha}^{2}}(\rho)\right\|-1\right), \quad \alpha=1, \cdots, m\right\} .
\end{aligned}
$$

From (79) and (84), it is seen that the lower bound for the class of mixed states with decompositions with respect to the generalized GHZ states is weaker than the one for the class of mixed states with decompositions with respect to the generalized $W$ states, in the sense that in (79) the realignment is associated with two arbitrary subsystems $\Theta_{1}$ and $\Theta_{2}$ such that $\Theta_{1} \cap \Theta_{2}=\varnothing$, but not necessary $\Theta_{1} \cup \Theta_{2}=\{1,2, \ldots, M\}$. While in (84) we simply treat the realignment associated with bipartite separations, so 
that the two subsystems $\Gamma_{\alpha}^{1}$ and $\Gamma_{\alpha}^{2}$ satisfy both $\Gamma_{\alpha}^{1} \cap \Gamma_{\alpha}^{2}=\varnothing$ and $\Gamma_{\alpha}^{1} \cup \Gamma_{\alpha}^{2}=\{1,2, \ldots, M\}$.

\subsubsection{Schmidt-correlated state}

The Schmidt-correlated (SC) states are the mixtures of pure states, sharing the same Schmidt bases. For any classical measurement related to the SC states, two observers will always obtain the same result [60]. Such SC states naturally appear in a bipartite system dynamics with additive integrals of motion [61].

An $M$-partite state $\rho$ in $C^{N} \otimes C^{N} \otimes \cdots \otimes C^{N}$ is called a Schmidt-correlated state if it can be expressed as

$$
\rho=\sum_{m, n=0}^{N-1} a_{m n}|m \cdots m\rangle\langle n \cdots n|
$$

where $\sum_{m=0}^{N-1} a_{m m}=1$. The SC state (85) can and can only be realized by an ensemble $\left\{p_{i},\left|\Phi_{i}\right\rangle\right\}, \quad\left|\Phi_{i}\right\rangle=$ $\sum \sqrt{a_{m m}} e^{i \Theta_{m}^{(i)}}|m \cdots m\rangle$, with $a_{m m}$ given in (85) [62].

Let $\operatorname{GHZ}(M, N)$ denote the $M$-partite maximally entangled state

$$
\begin{aligned}
\operatorname{GHZ}(M, N)= & \frac{1}{\sqrt{N}}(|0 \cdots 0\rangle+|1 \cdots 1\rangle \\
& +\cdots+|N-1, \cdots, N-1\rangle) .
\end{aligned}
$$

Then $\left|\Phi_{i}\right\rangle$ is equivalent to either a fully separable state or $\operatorname{GHZ}(M, t)(0<t \leqslant N)$ under stochastic local operation and classical communication (SLOCC) [63,64].

For a multipartite $\mathrm{SC}$ state $\rho$,

$$
\rho=\sum_{m, n=0}^{N-1} a_{m n}|m \cdots m\rangle\left\langle n \cdots n\left|=\sum_{i} p_{i}\right| \Psi_{i}\right\rangle\left\langle\Psi_{i}\right|,
$$

where $\left|\Psi_{i}\right\rangle$ takes the form $\left|\Psi_{i}\right\rangle=\sum_{m} c_{m}^{(i)}|m \cdots m\rangle$, $\sum_{m}\left|c_{m}^{(i)}\right|^{2}=1, a_{m n}=\sum_{i} p_{i} c_{m}^{(i)} c_{n}^{(i) *}$. It is easily seen that the concurrences of $\left|\Psi_{i}\right\rangle$ are the same for all reduced density matrices in bipartite decompositions. Due to the fact that $\rho_{1}=\operatorname{Tr}_{2 \cdots k}\left(\left|\Psi_{i}\right\rangle\left\langle\Psi_{i}\right|\right)=\sum_{m}\left|c_{m}^{(i)}\right|^{2}|m\rangle\langle m|$, one has

$$
\begin{aligned}
C\left(\left|\Psi_{i}\right\rangle\right) & =\sqrt{2\left(1-\sum_{m}\left|c_{m}^{(i)}\right|^{4}\right)}, \\
C(\rho) & =\min _{\left\{p_{i},\left|\Psi_{i}\right\rangle\right\}} \sum_{i} p_{i} \sqrt{2\left(1-\sum_{m}\left|c_{m}^{(i)}\right|^{4}\right)} \\
& =2 \min _{\left\{p_{i},\left|\Psi_{i}\right\rangle\right\}} \sum_{i} p_{i} \sqrt{\sum_{m<n}\left|c_{m}^{(i)} c_{n}^{(i)}\right|^{2}} .
\end{aligned}
$$

Taking into account that $\sum_{m}\left|c_{m}^{(i)}\right|^{2}=1$, one has $0 \leqslant C(\rho) \leqslant \sqrt{2\left(1-\frac{1}{N}\right)}$. For the state $\operatorname{GHZ}(M, N)$,
$C(\operatorname{GHZ}(M, N))=\sqrt{2\left(1-\sum_{m=0}^{N-1} \frac{1}{N^{2}}\right)}=\sqrt{2\left(1-\frac{1}{N}\right)}$.

Instead of bipartite decompositions, one may also directly use the concurrence formula Eq. (66) for multipartite states. Similarly, one can get $0 \leqslant C(\rho)$ $\leqslant \sqrt{m\left(1-\frac{1}{N}\right)}$ from the Lagrange multipliers method. Applying this to the state $\operatorname{GHZ}(M, N)$, one has similarly $C(\operatorname{GHZ}(M, N))=\sqrt{m\left(1-\frac{1}{N}\right)}$.

For general multipartite systems, one can deal with them as bipartite separations $\Gamma_{\alpha}^{1}$ and $\Gamma_{\alpha}^{2}$, which give rise to

$$
\begin{gathered}
1-\operatorname{Tr}\left(\left(\rho_{\Gamma_{\alpha}^{1}}\right)^{2}\right) \geqslant \frac{1}{D_{\alpha}\left(D_{\alpha}-1\right)} \max \left\{\left(\left\|\rho^{T_{\Gamma_{\alpha}^{1}}}\right\|-1\right)^{2},\right. \\
\left.\left(\left\|\mathcal{R}_{\Gamma_{\alpha}^{1} \mid \Gamma_{\alpha}^{2}}(\rho)\right\|-1\right)^{2}\right\}, \quad \alpha=1, \cdots, m,
\end{gathered}
$$

where $D_{\alpha}=\min \left(\operatorname{dim} \Gamma_{\alpha}^{1}, \operatorname{dim} \Gamma_{\alpha}^{2}\right), \operatorname{dim} \Gamma_{\alpha}^{1}\left(\operatorname{resp} . \operatorname{dim} \Gamma_{\alpha}^{2}\right)$ is the dimension associated with the subsystems contained in $\Gamma_{\alpha}^{1}\left(\operatorname{resp} . \Gamma_{\alpha}^{2}\right)$.

Therefore, for any $N_{1} \otimes N_{2} \otimes \cdots \otimes N_{M} \quad M$-partite mixed quantum state $\rho$, the concurrence $C(\rho)$ satisfies

$C(\rho) \geqslant K\left\{\max \left(\left\|\rho^{T_{\Gamma_{\alpha}^{1}}}\right\|,\left\|\mathcal{R}_{\Gamma_{\alpha}^{1} \mid \Gamma_{\alpha}^{2}}(\rho)\right\|\right)-1, \quad \alpha=1, \ldots, m\right\}$,

where $K=1 / \sqrt{D_{\alpha}\left(D_{\alpha}-1\right)}$.

Here, for general mixed states, it is difficult to find the relation between the concurrence of a pure state and the corresponding norm of the partial transposed state with respect to certain subsystems, like the one between (76) and (77). The bound (87) is obtained by bipartite separations of the system, and there is an extra factor $K$, which makes this bound weaker than (84), when it is applied to the special class of mixed states with decompositions with respect to the generalized $W$ states.

\section{Summary and conclusions}

We have given a review on the measures of quantum entanglement: entanglement of formation and concurrence. As it is difficult to calculate the EOF and concurrence for general mixed states due to the extremization involved in the calculation, analytic formulae for the EOF and concurrence are only obtained for a few special classes of mixed states. Fortunately, many strong separability criteria have been found. From these separability criteria many tight lower bounds of the EOF and concurrence have been obtained, which can detect, in particular, some bound entangled states. 


\section{References}

1. Nielsen M A, Chuang I L. Quantum Computation and Quantum Information. Cambridge: Cambridge University Press, 2000

2. Bennett C H, DiVincenzo D P, Smolin J A, et al. Mixed state entanglement and quantum error correction. Physical Review A, 1996, 54: 3824-3851

3. Horodecki M, Horodecki P, Horodecki R, et al. Classical capacity of a noiseless quantum channel assisted by noisy entanglement. Quantum Information and Computation, 2001, 1(3): 70-78

4. Bruß D. Characterizing entanglement. Journal of Mathematical Physics, 2002, 43: 4237

5. Plenio M B, Virmani S. An introduction to entanglement measures.Quantum Information and Computation, 2007, 7: 1-25

6. Uhlmann A. Fidelity and concurrence of conjugated states. A Physical Review A, 2000, 62: 032307

7. Albeverio S, Fei S M. A Note on Invariants and Entanglements. J Opt B: Quantum Semiclass Opt, 2001, 3: 223-227

8. Rungta P, Bužek V, Caves C M, et al. Universal state inversion and concurrence in arbitrary dimensions. Physical Review A, 2001, 64: 042315

9. Hill S, Wootters W K. Entanglement of a pair of quantum bits. Physical Review Letters, 1997, 78: 5022-5025

10. Wootters W K. Entanglement of formation of an arbitrary state of two qubits. Physical Review Letters, 1998, 80: 22452248

11. Osterloh A, Amico L, Falci G, et al. Scaling of entanglement close to a quantum phase transitions. Nature, 2002, 416: 608

12. Wu L A, Sarandy M S, Lidar D A. Quantum phase transitions and bipartite entanglement. Physical Review Letters, 2004, 93 : 250404

13. Ghosh S, Rosenbaum T F, Aeppli G, et al. Entangled quantum state of magnetic dipoles. Nature, 2003, 425: 48

14. Vedral V. Quantum physics. Entanglement hits the big time. Nature, 2003, 425: 28

15. Walborn S P, Souto Ribeiro P H, Davidovich L, et al. Experimental determination of entanglement with a single measurement. Nature, 2006, 440: 1022

16. Terhal B M, Vollbrecht K G H. The entanglement of formation for isotropic states. Physical Review Letters, 2000, 85: 2625-2628

17. Fei S M, Jost J, Li-Jost X Q, et al. Entanglement of formation for a class of mixed states. Physics Letters A, 2003, 310: 333-338

18. Fei S M, Li-Jost X Q. A class of special matrices and quantum entanglement. Reports on Mathematical Physics, 2004, 53: 195-210

19. Fei S M, Wang Z X, Zhao H. A note on entanglement of formation and generalized concurrence. Physics Letters A, 2004, 329: 414419

20. Rungta $\mathrm{P}$, Caves C M. I-concurrence and tangle for isotropic states. Physical Review A, 2003, 67: 012307

21. Chen P X, Liang L M, Li C Z, et al. A lower bound on entanglement of formation of $2 \otimes n$ system. Physics Letters A, 2002, 295: 175-177

22. Gerjuoy E. Lower bound on entanglement of formation for the qubit-qudit system. Physical Review A, 2003, 67: 052308

23. Òoziński A, Buchleitner A, Życzkowski K, et al. Entanglement of $2 \times K$ quantum systems. EurophysicsLetters, 2003, 62: 168

24. Audenaert K, Verstraete F, Moor B De. Variational characterisations of separability and entanglement of formation. Physical Review A, 2001, 64: 052304

25. Mintert F, Kuś M, Buchleitner A. Concurrence of mixed bipartite quantum states in arbitrary dimensions. Physical Review Letters, 2004, 92: 167902
26. Mintert F. Ph.D. thesis, Measures and dynamics of entangled states. Munich: Munich University, 2004

27. Chen K, Albeverio S, Fei S M. Concurrence of arbitrary dimensional bipartite quantum states. Physical Review Letters, 2005, 95: 040504

28. Chen K, Albeverio S, Fei S M. Entanglement of formation of bipartite quantum states. Physical Review Letters, 2005, 95: 210501

29. Breuer H P. Separability criteria and bounds for entanglement measures. Journal of Physics A: Mathematical and General, 2006, 39: 11847

30. Breuer H P. Optimal entanglement criterion for mixed quantum states. Physical Review Letters, 2006, 97: 080501

31. Vicente J I de. Lower bounds on concurrence and separability conditions. Physical Review A, 2007, 75: 052320

32. Zhang C J, Zhang Y S, Zhang S, et al. Optimal entanglement witnesses based on local orthogonal observables. Physical Review A, 2007, 76: 012334

33. Gao X H, Fei S M, Wu K. Lower bounds of concurrence of tripartite quantum systems. Physical Review A, 2006, 74: 050303(R)

34. Bennett C H, Bernstein H J, Popescu S, et al. Concentrating partial entanglement by local operations. Physical Review A, 1996, 53: 2046-2052

35. Werner R F. Quantum states with Einstein-Podolsky-Rosen correlations admitting a hidden-variable model. Physical Review A, 1989, 40: 4277-4281

36. Brun T A. Measuring polynomial functions of states. Quantum Information and Computation, 2004, 4: 401

37. Mintert F, Kus' M, Buchleitner A. Concurrence of mixed multi-partite quantum states. Physical Review Letters, 2005, 95: 260502

38. Fei S M, Li-Jost X. R-function related to entanglement of formation. Physical Review A, 2006, 73: 024302

39. Vollbrecht K G H, Werner R F. Entanglement measures under symmetry. Physical Review A, 2001, 64: 062307

40. Wootters W K. Entanglement of formation and concurrence. Quantum Information and Computation 1, 27(2001)

41. Chen K, Albeverio S, Fei S M. Concurrence-based entanglement measure for Werner states. Reports on Mathematical Physics, 2006, 58: 325-334

42. Albeverio S, Fei S M, Goswami D. Separability of Rank Two Quantum States. Physics Letters A, 2001, 286: 91-96

43. Fei S M, Gao X H, Wang X H, et al. Separability of rank two quantum states on multiple quantum spaces. Physics Letters A, 2002, 300: 555-562

44. Chen $\mathrm{K}, \mathrm{Wu} \mathrm{L}$ A. A matrix realignment method for recognizing entanglement. Quantum Information and Computation, 2003, 3: 193-202

45. Horodecki M, Horodecki P, and Horodecki R. Separability of mixed quantum states: linear contractions approach. Open Systems and Information Dynamics, 2006, 13: 103

46. Rudolph O. Further results on the cross norm criterion for separability. Quantum Information Processing, 2005, 4: 219

47. Ou Y C, Fan H, Fei S M. Concurrence, distillability, and distributed entanglement for arbitrary quantum states. arXiv:0711.2865v2, 2007

48. Horodecki M, Horodecki P. Reduction criterion of separability and limits for a class of distillation protocols. Physical Review A, 1999, 59: 4206-4216

49. Vollbrecht K G H, Werner R F. Entanglement measures under symmetry. Physical Review A, 2001, 64: 062307

50. Hofmann H F, Takeuchi S. Violation of local uncertainty relations as a signature of entanglement. Physical Review A, 2003, 68: 032103 
51. Hofmann H F. Bound entangled states violate a nonsymmetric local uncertainty relation. Physical Review A, 2003, 68: 034307

52. Gühne O, Mechler M, Toth G, et al. Entanglement criteria based on local uncertainty relations are strictly stronger than the computable cross norm criterion. Physical Review A, 2006, 74: 010301(R)

53. Yu S X, Liu N L. Entanglement detection by local orthogonal observables. Physical Review Letters, 2005, 95: 150504

54. Vicente J I de. Separability criteria based on the Bloch representation of density matrices. Quantum Information and Computation, 2007, 7: 624

55. Bennett $\mathrm{C} \mathrm{H}$, DiVincenzo D P, Mor $\mathrm{T}$, et al. Unextendible Product bases and bound entanglement. Physical Review Letters, 1999, 82: 5385-5388

56. Fei S M, Li-Jost X, Sun B Z. A Class of Bound Entangled States. Physics Letters A, 2006, 352: 321

57. Albeverio S, Chen K, Fei S M. Generalized reduction criterion for separability of quantum states. Physical Review A, 2003, 68: 062313
58. Horn R A, Johnson C R. Topics in Matrix Analysis. New York: Cambridge University Press, 1991

59. Acin A, Andrianov A, Costa L, et al. Generalized Schmidt decomposition and classification of three-quantum-bit states. Physical Review Letters, 2000, 85: 1560-1563

60. E.M. Rains. Bound on distillable entanglement.Physical Review A, 1999, 60: 179

61. M. Khasin, R. Kosloff. Rise and fall of quantum and classical correlations in opensystem dynamics. Physical Review A, 2007, 76: 012304

62. Zhao M J, Fei S M, Wang Z X. Entanglement of Multipartite Schmidt-correlated States. Physics Letters A, 2008, 372: 25522557

63. Pan F, Lu G Y, Draayer J P. Classification and quantification of entangled bipartite qutrit pure states. International Journal of Modern Physics B, 2006, 20: 1333-1342

64. Lamata L, León J, Salgado D, et al. Inductive classification of multipartite entanglement under SLOCC. Physical Review A, 2006, 74: 052336 